\title{
Targeting inflammatory monocytes in sepsis-associated encephalopathy and long-term cognitive impairment
}

\author{
Graciela Andonegui, ${ }^{1,2}$ Erin L. Zelinski, ${ }^{3}$ Courtney L. Schubert, ${ }^{1,2}$ Derrice Knight, ${ }^{1}$ Laura A. Craig, ${ }^{4}$ \\ Brent W. Winston, ${ }^{1,2,5,6}$ Simon C. Spanswick, ${ }^{4}$ Björn Petri, ${ }^{7,8}$ Craig N. Jenne, ${ }^{1,2,7}$ Janice C. Sutherland, ${ }^{3}$ \\ Rita Nguyen, ${ }^{1}$ Natalie Jayawardena, ${ }^{1}$ Margaret M. Kelly, ${ }^{9,10,11}$ Christopher J. Doig, ${ }^{2,5,12}$ \\ Robert J. Sutherland, ${ }^{3}$ and Paul Kubes ${ }^{1,11}$ \\ IImmunology Research Group, Calvin, Phoebe and Joan Snyder Institute for Chronic Diseases, and 2Department of \\ Critical Care Medicine, Cumming School of Medicine, University of Calgary, Calgary, Alberta, Canada. ${ }^{3}$ Canadian Centre \\ for Behavioural Neuroscience, Department of Neuroscience, University of Lethbridge, Lethbridge, Alberta, Canada. \\ ${ }^{4}$ Regeneration Unit in Neurobiology Facility, Hotchkiss Brain Institute, ${ }^{5}$ Department of Medicine, ${ }^{6}$ Department of \\ Biochemistry and Molecular Biology, ${ }^{7}$ Department of Microbiology, Immunology and Infectious Diseases, ${ }^{8}$ Mouse \\ Phenomics Resource Laboratory, Snyder Institute for Chronic Diseases, ${ }^{9}$ Airway Inflammation Research Group, Calvin, \\ Phoebe and Joan Snyder Institute for Chronic Diseases, ${ }^{10}$ Department of Pathology and Laboratory Medicine, "Department \\ of Physiology and Pharmacology, and ${ }^{12}$ Department of Community Health Sciences, Cumming School of Medicine, \\ University of Calgary, Calgary, Alberta, Canada.
}

\begin{abstract}
Sepsis-associated encephalopathy manifesting as delirium is a common problem in critical care medicine. In this study, patients that had delirium due to sepsis had significant cognitive impairments at 12-18 months after hospital discharge when compared with controls and Cambridge Neuropsychological Automated Test Battery-standardized scores in spatial recognition memory, pattern recognition memory, and delayed-matching-to-sample tests but not other cognitive functions. A mouse model of $S$. pneumoniae pneumonia-induced sepsis, which modeled numerous aspects of the human sepsis-associated multiorgan dysfunction, including encephalopathy, also revealed similar deficits in spatial memory but not new task learning. Both humans and mice had large increases in chemokines for myeloid cell recruitment. Intravital imaging of the brains of septic mice revealed increased neutrophil and CCR2 ${ }^{+}$inflammatory monocyte recruitment (the latter being far more robust), accompanied by subtle microglial activation. Prevention of CCR2+ inflammatory monocyte recruitment, but not neutrophil recruitment, reduced microglial activation and other signs of neuroinflammation and prevented all signs of cognitive impairment after infection. Therefore, therapeutically targeting CCR2+ inflammatory monocytes at the time of sepsis may provide a novel neuroprotective clinical intervention to prevent the development of persistent cognitive impairments.
\end{abstract}

Authorship note: GA and ELZ contributed equally to this work. CJD, $\mathrm{RJS}$, and PK contributed equally to this work.

Conflict of interest: The authors have declared that no conflict of interest exists.

Submitted: December 19, 2017

Accepted: April 4, 2018

Published: May 3, 2018

\section{Reference information:}

JCI Insight. 2018;3(9):e99364. https://doi.org/10.1172/jci.

insight.99364.

\section{Introduction}

A recent consensus has defined sepsis as a life-threatening organ dysfunction caused by a dysregulated host response to infection (1). Sepsis is the most common cause of death in hospitalized patients in Canada and the US, causing $11 \%$ of the deaths in hospitals per year (2). Community-acquired pneumonia is the most prevalent cause of sepsis, accounting for $50 \%$ of these hospitalized patients (3), and Streptococcus pneumoniae (S. pneumoniae), a Gram-positive bacterium, is the most common cause of this condition $(4,5)$. Although the mortality rate of sepsis has decreased over the past 15 years $(6)$, the overall numbers of patients with sepsis has increased.

Sepsis-associated encephalopathy (SAE), a diffuse brain dysfunction secondary to infections outside the central nervous system, develops in approximately $70 \%$ of patients with sepsis (7). Sepsis survivors demonstrate long-term cognitive impairment, including alterations in memory, attention, and concentration, and many are unable to return to their previous employment (8-11). The mechanisms causing SAE or delirium are unclear and understudied. In the absence of an understanding of its cause, treatment is 
limited to nonspecific "symptom" management, such as patient sedation, and is clearly unsatisfactory. Direct pathogen dissemination into the brain during sepsis is very uncommon and, therefore, other factors must contribute. Our hypothesis is that the inappropriate activation of the immune system and cerebral leukocyte recruitment results in brain inflammation that contributes to the development of SAE and leads to long-term cognitive impairments. As such, we tested whether inflammation of the brain microvasculature leads to cerebral dysfunction (blood-brain barrier breakdown) and long-term cognitive impairments.

The hallmark of inflammation is the recruitment of inflammatory leukocytes. Neutrophils are the exemplar immune cells mediating much of the tissue injury associated with various inflammatory processes. This is primarily based on the fact that neutrophils are the main leukocytes recruited to the inflamed foci in response to infection or tissue injury and that they produce potent oxidants, release proteases, and form toxic neutrophil extracellular traps (12). During a systemic inflammatory response to an infection there is excessive or inappropriate neutrophil infiltration into different tissues (13). Consequently, the role of the neutrophil has dominated attention in sepsis research. Neutrophil recruitment into the brain requires an initial tethering of the immune cell to the endothelium via selectins and subsequent activation by chemokines, such as IL-8 (KC and MIP-2 in mice), acting through the chemokine receptor CXCR2 on neutrophils, resulting in integrin activation and firm adhesion (14). The neutrophils have then been shown to transmigrate across the endothelial barrier, causing vascular barrier breakdown (15). In addition to neutrophils, other immune cells, such as inflammatory monocytes, are also recruited, although their specific role remains less clear (16). Inflammatory monocytes also use integrins for recruitment; however, a major difference from neutrophils is their use of the chemokine receptor CCR2 for activation and subsequent adhesion to the vessel wall. Various subsets of monocytes have been identified based on Ly6C and chemokine receptor expression. Monocytes with Ly6C ${ }^{\text {hi }} C C R 2{ }^{\text {hi }} C X 3 C R 1^{\text {lo }}$ expression are referred to as the inflammatory monocyte subset $(17,18)$. A second subset of $\mathrm{Ly} 6 \mathrm{C}^{\mathrm{lo}} \mathrm{CCR} 2{ }^{\text {lo }} \mathrm{CX} 3 \mathrm{CR} 1^{\text {hi }}$ monocytes is found patrolling various tissues but does not overtly cause inflammation (18). A second subset of monocytes is Ly6C ${ }^{\text {lo }}, \mathrm{CCR} 2^{\text {lo }}$, and CX3CR1 ${ }^{\text {hi }}$, and this subset is found patrolling various tissues but not overtly causing inflammation (18).

In this study, patients that recovered from sepsis performed poorly in behavioral tests that require the integrity of the medial temporal lobe and associated cortical regions at 12-18 months after recovery. A mouse model of $S$. pneumoniae pneumonia-induced sepsis replicated these long-term cognitive deficits and had an increase in neutrophil and CCR2 ${ }^{+}$inflammatory monocyte recruitment and microglial activation at 24 hours after infection. Our data show that inflammatory monocyte, but not neutrophil recruitment, was found to induce the hallmarks of inflammation and cause the long-term cognitive impairment associated with acute sepsis.

\section{Results}

Patients recovered from sepsis show signs of cognitive impairment. Eleven patients that had documented delirium during sepsis were assessed 12-18 months after intensive care unit (ICU) hospital discharge using a series of cognitive tests. Table 1 shows the demographic and clinical characteristics of the enrolled patients. In these patients, the lungs were the primary focus of infection. Compared with control participants, sepsis-recovered patients had significantly impaired performance on pattern recognition memory, impaired spatial recognition memory, and delayed matching to sample tasks $(P<0.05$; Figure $1, \mathrm{~A}-\mathrm{C})$. Trends were also observed for the paired associates learning $(P=0.06)$ and spatial span $(P=0.06)$ task performance compared with controls and Cambridge Neuropsychological Automated Test Battery standardized scores (Supplemental Figure 1, A and B; supplemental material available online with this article; https://doi. org/10.1172/jci.insight.99364DS1). It is also important to note that there were a number of tests that were not significantly different, including motor screen, spatial working memory, stockings of Cambridge, big little circle, and intradimensional/extradimensional set shift task, suggesting specific cognitive impairments in visuospatial memory function, not overall neurocognitive function (Supplemental Figure 1, C and D; and Supplemental Figure 2, A-G). The pattern of impairment observed among sepsis survivors was indicative of possible medial temporal and restricted frontal lobe dysfunction and, more specifically, dysfunction of the parahippocampal complex, which is exquisitely sensitive to environmental perturbations.

Cytokine profile in septic patients. Forty-eight cytokines and chemokines were assessed in the sera of septic patients. Serum_samples, 16 from ICU controls and 34 from septic patients, were retrospectively selected from the ICU tissue bank to study the cytokine profile. Table 2 shows the demographic and 
A Pattern Recognition Memory

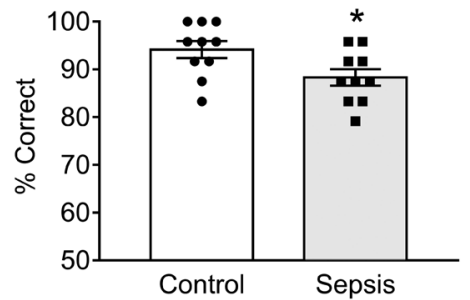

B

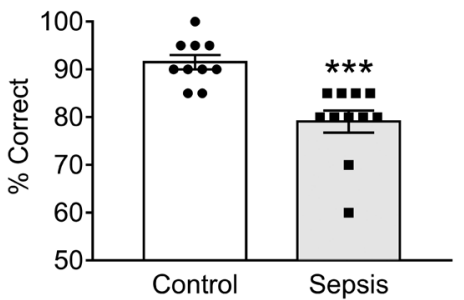

C Delayed Match to Sample

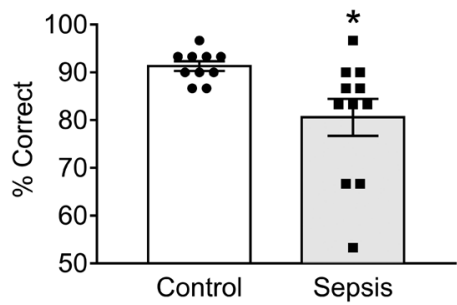

D

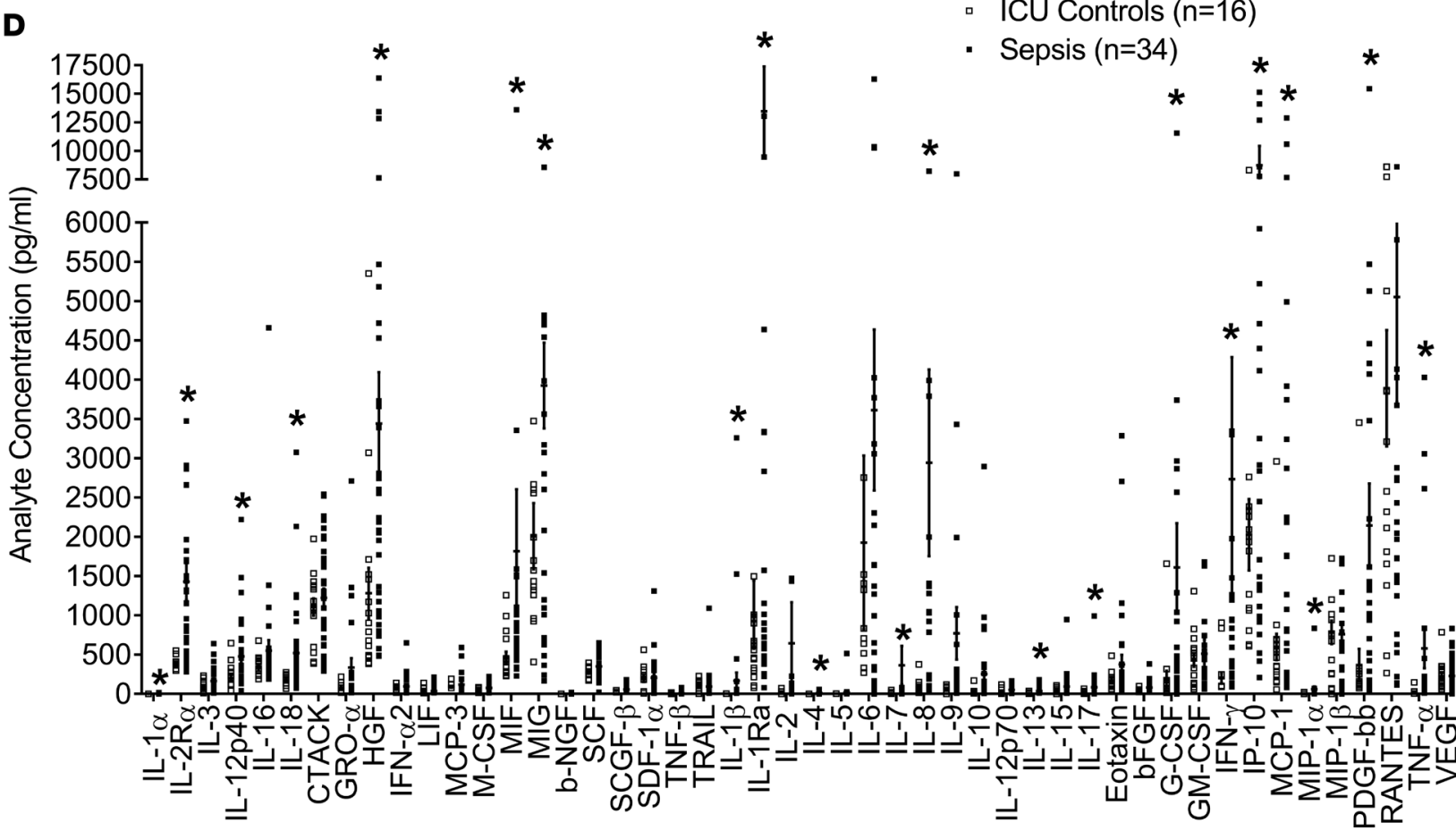

Figure 1. Behavioral tests and serum cytokines levels in ICU patients. ICU sepsis survivors were evaluated at 12 months after hospital discharge in (A) pattern recognition memory (control $n=10$, sepsis $n=11$ ), (B) spatial recognition memory (control $n=10$, sepsis $n=10$ ), and (C) delayed match to sample (control $n=10$, sepsis $n=11$ ). Data represent mean \pm SEM. ${ }^{*} P<0.05$, ${ }^{* * *} P<0.001$ vs. controls, unpaired 2-tailed $t$ test. (D) The level of blood cytokines was determined at 24 hours after hospital admission in ICU controls $(n=16)$ and septic patients $(n=34)$. Data represent mean \pm SEM. ${ }^{*} P<0.05$ vs. ICU controls, Mann-Whitney $U$ test.

clinical characteristics of all patient samples studied. A significant increase in IL-1 $\alpha$, IL-2R $\alpha$, IL-12p40, IL-18, HGF, MIF, MIG, IL-1 $\beta$, IL-1Ra, IL-4, IL-7, IL-8, IL-13, IL-17, G-CSF, IFN- $\gamma$, IP-10, MCP-1, MIP-1 $\alpha$, PDGF-bb, and TNF- $\alpha$ was observed in septic patients at 24 hours after hospital admission (Figure 1D) versus ICU controls. Worth noting is the large increase in the antiinflammatory IL-1Ra and chemokines for neutrophils (IL-8) but also for other immune cells, including monocytes (MCP-1).

$S$. pneumoniae pneumonia-induced sepsis mouse model. Intratracheal administration of $S$. pneumoniae into the lungs of mice resulted in pneumonia and sepsis, including increased gut permeability and decreased cardiac output as previously described (19). We could detect almost no bacteria in blood and no bacteria in brain but significant bacteria in lungs at 24 hours (19). This is entirely consistent with human sepsis, in which cerebral bacteria are extremely rare (7). In the absence of bacteria in the brain, we postulated that inflammatory mediators might induce brain inflammation $(20,21)$. S. pneumoniae infection induced increases in mediators at 4 hours that reached peak levels by 24 hours. The levels of several cytokines are shown in Figure 2, A-H. A significant increase in KC (homologous to IL-8 in humans), G-CSF, MCP-1, MIP-1 $\alpha$, and IP-10 was found at 24 hours after infection. A trend was observed for TNF- $\alpha(P=0.06)$. Interestingly, the same proinflammatory molecules were significantly increased in the blood of septic patients 


\section{Table 1. Demographic and clinical characteristics of Lethbridge patients}

\begin{tabular}{|c|c|}
\hline Characteristics & Sepsis patients \\
\hline No. of patients & 11 \\
\hline Male/Females ( $n$ ) & $4: 7$ \\
\hline Age (yr) & $53(33-67)$ \\
\hline APACHE II (admission) & $17(12-27)$ \\
\hline SOFA (day 1) & $8(7-11)$ \\
\hline \multirow[t]{3}{*}{ Primary ICNARC code $(n)$} & Septic shock (1) \\
\hline & Pneumonia (9) \\
\hline & Ascending cholangitis (1) \\
\hline Length of ICU stay (d) & $11(6-18)$ \\
\hline Patients with organ insufficiency ( $n$ ) & 2 \\
\hline \multirow[t]{2}{*}{ Primary focus of infection ( $n$ ) } & Lung (9) \\
\hline & Gastrointestinal (2) \\
\hline \multirow[t]{2}{*}{ Confirmed infection $(n)$} & Gram-positive bacteria (3) \\
\hline & Gram-negative bacteria (3) \\
\hline Deaths $(n)$ & 0 \\
\hline \multicolumn{2}{|c|}{$\begin{array}{l}\text { Primary Intensive Care National Audit and Research Centre (ICNARC) code, acut } \\
\text { physiology and chronic health evaluation (APACHE), and sequential organ failur } \\
\text { assessment (SOFA) scores were assessed upon admittance to the intensive care } \\
\text { unit. All data are median (interquartile range) unless otherwise noted. }\end{array}$} \\
\hline
\end{tabular}

at 24 hours after hospital admission (Figure 1D). Surprisingly, however, only KC and G-CSF were increased in the CSF (Figure 2, B and D).

S. pneumoniae pneumonia-induced sepsis is associated with long-term cognitive impairment. S. pneumoniae-infected wildtype mice show decreased movement and lethargy at 4 hours after infection, and these signs become worse at 12 hours after infection; if the mice survive past 36 hours, their general behavior recovered to a healthy state by 48 hours (19). We conducted a battery of behavioral tests to evaluate if $S$. pneumoniae pneumonia-induced sepsis was associated with long-term cerebral impairment. Mice were infected and allowed to recover for at least 14 days (postinfection mice). These mice performed similarly to control mice when tested in open-field, Y-maze, and elevated plus maze behavioral tests (Supplemental Figure 3). The Morris water maze test has a 3-day learning component, followed by a memory test component. There was no impairment in learning during the training session for 3 days; both control and postinfection mice learned the task equivalently, as shown by the path length and latency time in finding the platform (Figure 2, I and $\mathrm{J}$ ). However, on the fourth day, when the platform was removed from the pool, postinfection mice traveled signifi-

cantly less distance and spent less time in the target quadrant where the platform had been located (Figure 2, $\mathrm{K}$ and $\mathrm{L}$ ), showing a deficit in spatial memory.

We further assessed the duration of this cognitive impairment and found that it persisted at all times observed. For example, the water maze test was assessed again at 9 weeks after infection in a different pool and room. Both groups of mice learned the task in the same way, as shown by similar path lengths (Figure 3A); however, when tested on the fourth day (67 days after infection), postinfection mice traveled significantly less distance in the target quadrant, denoting that the deficit in spatial memory is a long-term impairment (Figure 3B). Behavioral tests specifically designed to evaluate the functional integrity of hippocampus and amygdala were performed to determine which part of the brain was involved in the long-term memory impairment $(22,23)$. Mice that had recovered from infection exhibited a lower freezing response in the immediate shock deficit test (Figure 3C) and contextual fear conditioning test (Supplemental Figure 4), tests designed to examine hippocampal function. These differences were observed as late as 18 weeks after infection recovery. By contrast, in the cued fear conditioning test (Figure 3D), which examines amygdala function, both control and postinfection mice froze with similar frequency.

To determine whether there were overt signs of injury, MRI was performed in the mice that had recovered from infection (Figure 3E) and noted no differences in hippocampal volume compared with control mice (Figure $3 \mathrm{~F}$ ), suggesting that there was no obvious dysfunction that translated in anatomical changes in the hippocampus. In addition, histology of the hippocampus section did not show any changes in the cellularity of the dentate gyrus region between controls and mice that had recovered from infection (Supplemental Figure 5). However, when we assessed signs of brain inflammation/dysfunction, there was an increased blood-brain barrier permeability at 24 hours after infection (Figure 3, G and $\mathrm{H}$ ). The value was comparable to the blood-barrier permeability increase triggered by $B$. pertussis toxin, our most potent positive control, suggesting potential inflammation at early time points. As such, we performed cerebrovascular microscopy to further examine the state of inflammation.

S. pneumoniae pneumonia-induced sepsis is associated with increased neutrophil recruitment into the brain. Increased $\mathrm{KC}$ in mice and IL-8 in humans prompted us to examine neutrophil recruitment in the brain microcirculation. Intravital microscopy on mice infected with $S$. pneumoniae showed a significant increase in neutrophil rolling flux and adhesion compared with control mice in the brain vasculature of the frontal cortex at 24 hours after infection (Figure 4). Under basal conditions, no neutrophils were seen interacting with brain microvessels (Figure $4 \mathrm{~A}$ ), whereas following $S$. pneumoniae infection, a significant $(P<0.001)$ increase was noted (Figure 4B). At least 3-4 neutrophils could be seen rolling per minute (Figure $4 \mathrm{C}$ ) and 
A

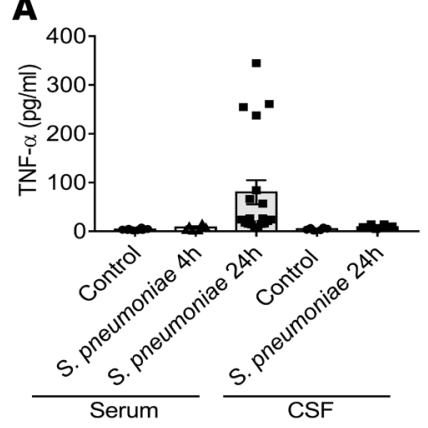

E

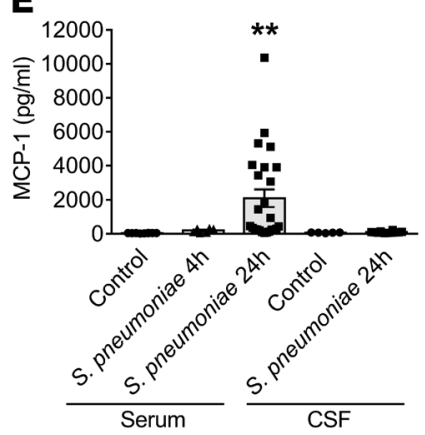

I

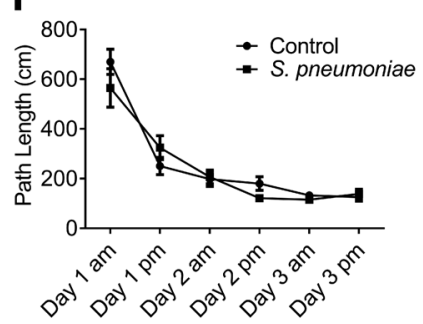

B

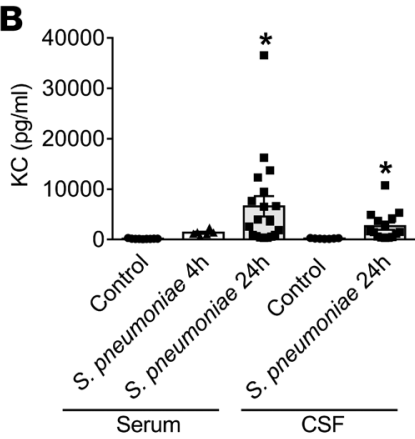

$\mathbf{F}$

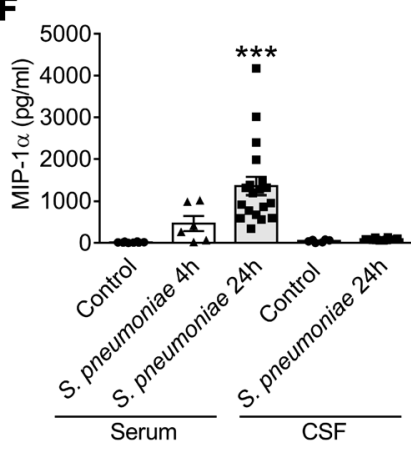

J

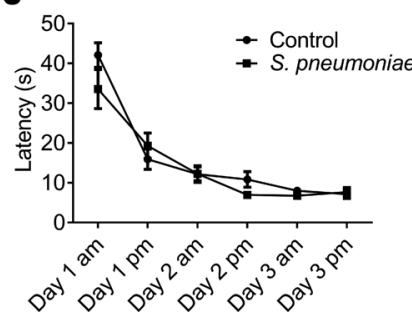

C

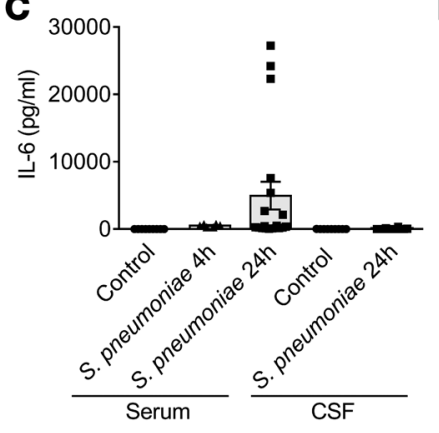

G

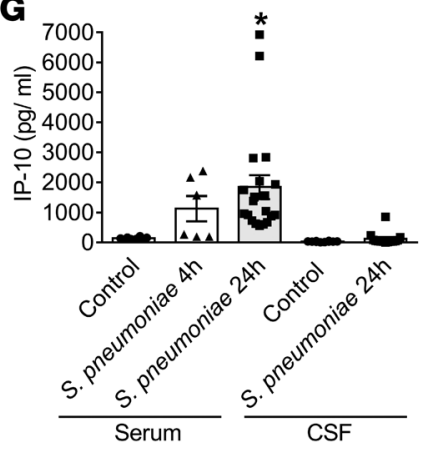

$\mathbf{K}$

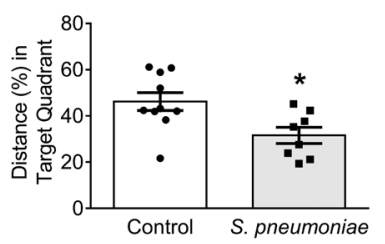

D

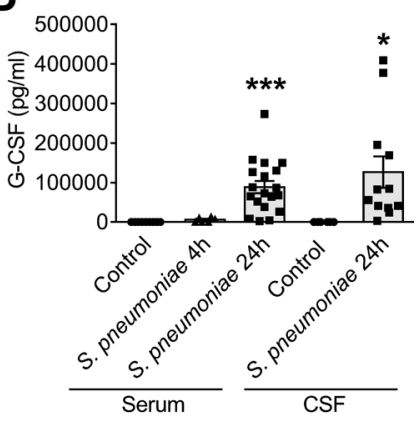

H

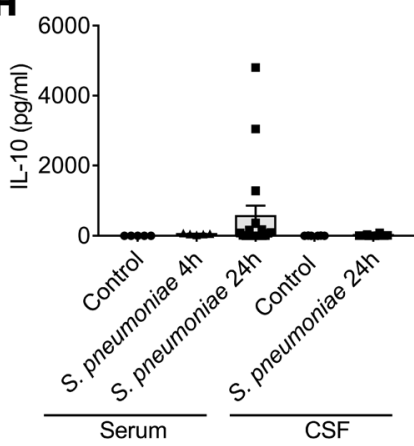

$\mathbf{L}$

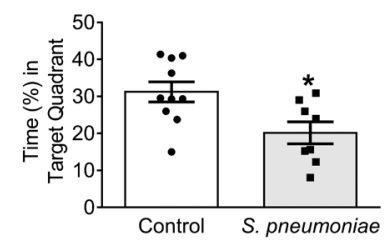

Figure 2. S. pneumoniae-infected mice have increased blood and cerebrospinal fluid cytokines. Mice were infected with S. pneumoniae, and blood was extracted at 4 hours and 24 hours (S. pneumoniae $4 \mathrm{~h}$ or $24 \mathrm{~h}$ serum) or cerebrospinal fluid (CSF) was extracted at 24 hours after infection to measure a large cytokine array. Several cytokines are shown in (A) TNF- $\alpha$, (B) KC, (C) IL-6, (D) G-CSF, (E) MCP-1, (F) MIP-1 $\alpha$, (C) IP-10 levels, and (H) IL-10. Data in A-H represent mean \pm SEM of $n=5-10$ in blood serum control, $n=5-6$ in blood serum S. pneumoniae 4 hours, $n=18-20$ in blood serum S. pneumoniae 24 hours, $n=5-10$ in CSF control, and $n=12-15$ in CSF S. pneumoniae. ${ }^{*} P<0.05,{ }^{* *} P<0.01,{ }^{* *} P<0.001$ vs. control, 1-way ANOVA followed by Dunnett's multiple comparison's test for the blood serum samples and unpaired $t$ test for the CSF samples. Mice recovered from infection showed cognitive impairment in Morris water maze. Mice were infected with S. pneumoniae and allowed to recover for 2 weeks and, at this time, the Morris water maze test was assessed (see Methods). (I) Path length during training, (J) latency during training, (K) percentage of distance traveled in the target quadrant, and (L) percentage of time in target quadrant both on the fourth day after the platform was removed and the mouse memory strength were evaluated. Data in I-L represent mean \pm SEM of $n=10$ in control and $n=8$ in S. pneumoniae-infected mice group. ${ }^{*} P<0.05$ vs. control mice, unpaired 2-tailed $t$ test.

adhering per $100 \mu \mathrm{m}$ (Figure 4D) in the cerebrovasculature. Figure 4E shows neutrophils in a coronal brain section of the hippocampus. Neutrophils were found throughout the whole coronal brain section at 24 hours after infection (Figure 4F), and these cells all remained in the vasculature. Anti-KC mAb administered at 4 hours after infection did not affect neutrophil rolling flux (Figure 5A) but significantly decreased neutrophil adhesion in the brain vasculature (Figure $5 \mathrm{~B}$ ) and reduced neutrophil counts in coronal sections (Figure $5 \mathrm{C}$ ). However, postinfection mice treated with anti-KC mAb still had memory deficits, assessed as decreased travel distance in the target quadrant (Figure 5D). To ensure that 4 hours after the start of infection was not too late for treatment with anti-KC mAb, $\mathrm{CXCR} 2^{-/-}$mice (mice lacking the $\mathrm{KC}$ receptor) were tested and also showed a cognitive impairment trend (data not shown).

The possibility exists that KC inhibition delayed neutrophil influx into the lungs and made the infection worse. Therefore, in a second set of experiments, we treated mice with anti-P selectin mAb immediately after infection to prevent brain neutrophil recruitment without affecting neutrophil recruitment in the lungs as previously reported (13). Anti-P selectin $\mathrm{mAb}$ treatment significantly inhibited neutrophil rolling flux and adhesion in the brain (Figure 5, A and B) and reduced neutrophil numbers in the coronal sections (Figure 5C). 
Table 2. Demographic and clinical characteristics of the enrolled patients

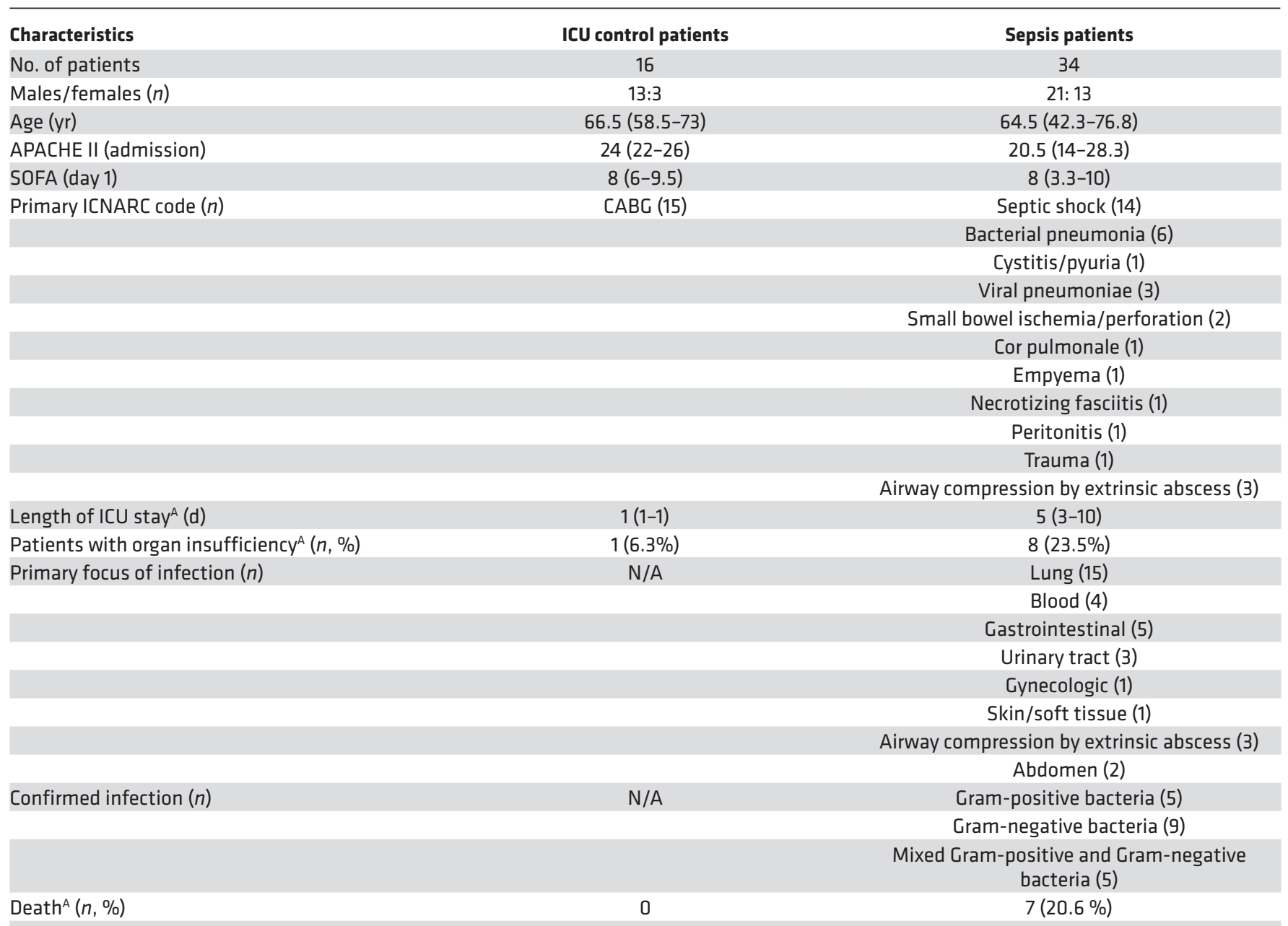

${ }^{A}$ Statistically significant feature $(P<0.05)$. Primary Intensive Care National Audit and Research Centre (ICNARC) code, acute physiology and chronic health evaluation (APACHE), and sequential organ failure assessment (SOFA) scores were assessed upon admittance to intensive care unit (ICU). All data are median (interquartile range) unless otherwise noted. CABG, coronary artery bypass surgery.

Surprisingly, despite complete inhibition of neutrophil recruitment following anti-P selectin mAb treatment, memory impairment was noted (Figure 5D). These data suggest that neutrophil recruitment to the brain is not responsible for the long-term cognitive impairment observed in postinfection mice.

S. pneumoniae pneumonia-induced sepsis is associated with an increase in CCR2 inflammatory monocyte recruitment into the brain. Intriguingly, MCP-1, a monocyte chemokine and the ligand for CCR2 was greatly elevated in both mice (Figure 2E) and humans (Figure 1D). Using fluorescent reporter $\left(\mathrm{CX} 3 \mathrm{CR} 1^{\mathrm{GFP} / \mathrm{WT}} \mathrm{CCR} 2^{\mathrm{RFP} / \mathrm{WT}}\right)$ mice that report for both inflammatory and patrolling monocytes revealed a significant increase in rolling and adhesion of CCR2 $2^{+}$inflammatory monocytes (RFP positive, visualized in red) but not patrolling monocytes (GFP positive, visualized in green) at 24 hours after infection (Figure 6, A-D). In the coronal brain sections, we also observed an increase in CCR2 ${ }^{+}$ inflammatory monocyte recruitment at 24 hours after infection (Figure 6, E-G). Monocytes were distributed evenly throughout the stitched image of the entire brain slice. Unexpectedly, the increase in $\mathrm{CCR}^{+}{ }^{+}$inflammatory monocyte recruitment was 3 times greater than the neutrophil recruitment at 24 hours after infection. It is worth mentioning that by 72 hours the number of monocytes and neutrophils was greatly decreased and by 2 weeks we observed no cells trafficking through the cerebrovasculature, just like in uninfected mice. 
A

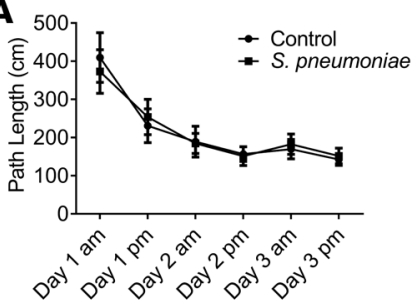

C

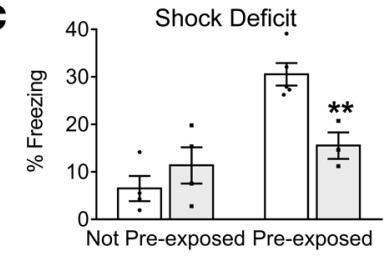

- Control

E

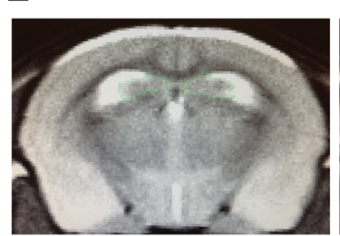

F

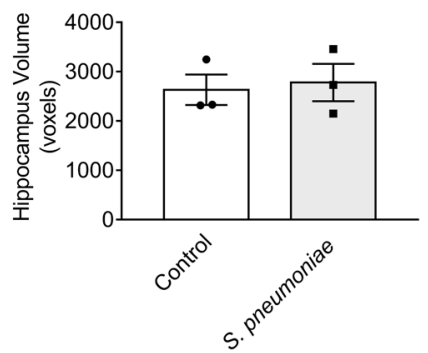

B
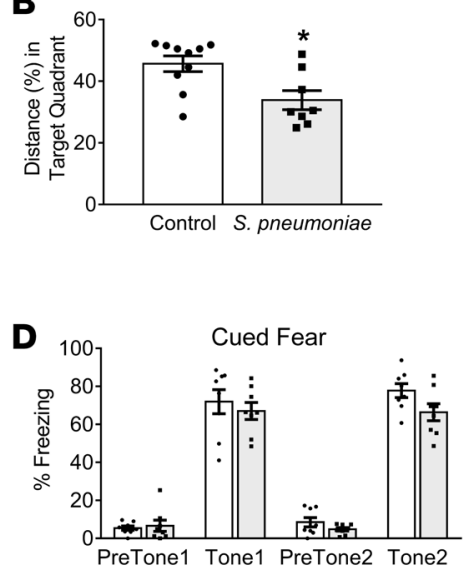

- S. pneumoniae
H

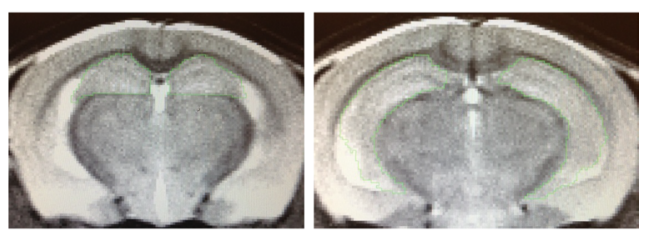

G

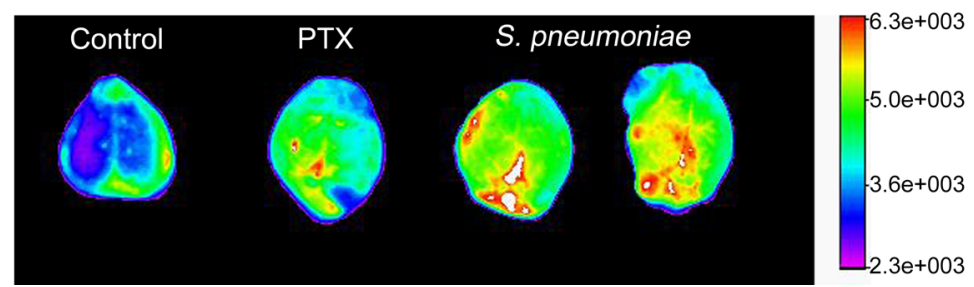

Figure 3. Morris water maze at $\mathbf{9}$ weeks after infection. Mice were infected with S. pneumoniae and allowed to recover for 2 weeks. At this time, the Morris water maze was assessed. Then, at 9 weeks after infection, the Morris water maze was assessed again in a different pool and different room. (A) Path length during the learning days. (B) Percentage of distance spent in the target quadrant on the fourth day when platform was removed to assess memory strength in mice. Data in $\mathbf{A}$ and $\mathbf{B}$ represent mean \pm SEM of $n=10$ in control and $n=8$ in S. pneumoniae recovered from infection. ${ }^{*} P<0.05$ vs. control mice, unpaired 2-tailed $t$ test. Hippocampus but not amygdala function is altered in mice recovered from S. pneumoniae infection. Mice were infected with S. pneumoniae for 18 weeks, and, at this time, the behavioral tests were conducted. (C) Immediate shock deficit contextual freezing. (D) Cued fear conditioning data in $\mathbf{C}$ and $\mathbf{D}$ represent mean \pm SEM of $n=10$ in control and $n=8$ in $S$. pneumoniae-infected mice. ${ }^{*} P<0.01$ vs. control mice, 2-way ANOVA followed by Sidak's multiple comparisons test. MRI brain images of mice recovered from S. pneumoniae infection. (E) Representative multislice images of a mouse brain after recovery from $S$. pneumoniae infection at 18 weeks after infection. (F) Hippocampus volume was assessed by analyzing voxel based segmentation of the MRI stacks. Data represent mean \pm SEM of $n=3$. Effects of S. pneumoniae on blood-brain barrier permeability. Mice were treated with S. pneumoniae for 24 hours, and, at this time, permeability was assessed with Evans Direct Blue. (G) Quantification of brain permeability. Data represent mean \pm SEM of $n=3 .{ }^{*} P<0.01$ vs. control mice, 1-way ANOVA followed by Tukey's multiple comparisons test. (H) Permeability image in control, B. pertussis toxin- (PTX, used as a positive control for permeability), and S. pneumoniae-infected mice for 24 hours.

$C C R 2^{\text {def }}$ mice show decreased monocyte recruitment after $S$. pneumoniae infection. CCR2 ${ }^{\text {def }}\left(\mathrm{CX} 3 \mathrm{CR} 1^{\mathrm{GFP} / \mathrm{WT}} \mathrm{C}\right.$ $\mathrm{CR} 2^{\mathrm{RFP} / \mathrm{RFP}}$ ) mice lack circulating inflammatory monocytes and, as such, showed a very significant decrease in CCR2 $2^{+}$inflammatory monocyte rolling flux and adhesion in the brain vasculature at 24 hours after infection (Figure 7, A and B). This was not compensated for by an increase in CX3CR $1^{+}$monocytes. Coronal sections showed a significant decrease in $\mathrm{CCR} 2^{+}$inflammatory monocyte adhesion throughout the brain compared with infected wild-type mice (Figure 7, C and D). CCR2 ${ }^{\text {def }}$ mice that had recovered from infection learned the water maze the same way as control mice (data not shown) and had no cognitive impairment whatsoever (Figure 7, E and F). Interestingly, CCR2 ${ }^{\text {def }}$ mice showed a significant increase in blood-brain barrier permeability at 24 hours after infection, as observed in infected WT mice (Supplemental Figure 6), suggesting that this dysfunction does not correlate with the cognitive impairment. Thus, these data suggest that CCR2 ${ }^{+}$ inflammatory monocytes play an important role in the cognitive impairment associated with $S$. pneumoniae pneumonia-induced sepsis but not as a result of increased vascular permeability.

Anti-CCR $2 \mathrm{mAb}$ treatment prevents cognitive impairment. The effect of treatment of wild-type mice with an anti-CCR $2 \mathrm{mAb}$ before infection was evaluated. Anti-CCR $2 \mathrm{mAb}$ treatment significantly abolished CCR2 ${ }^{+}$ 
A

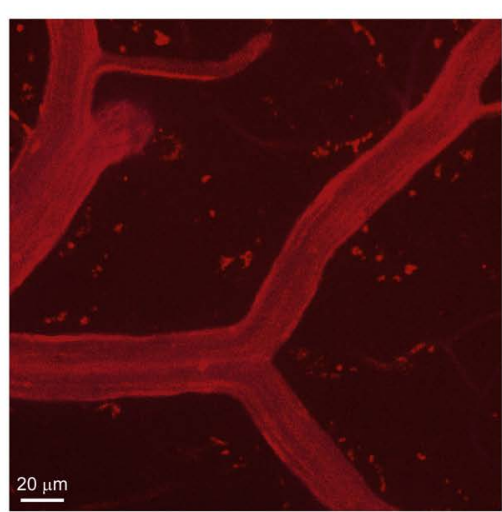

C

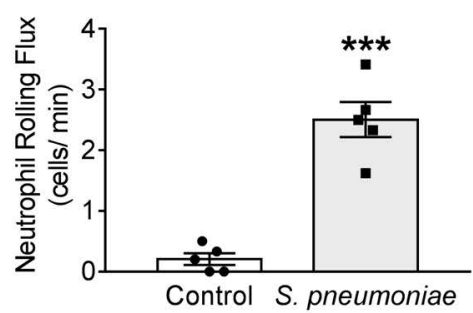

E

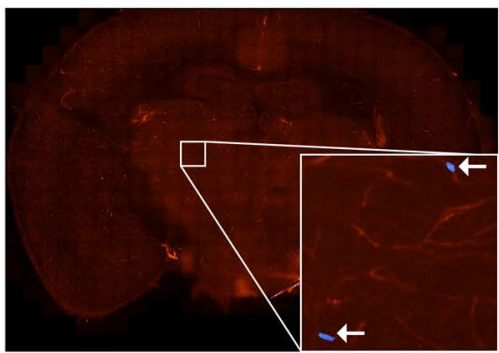

B

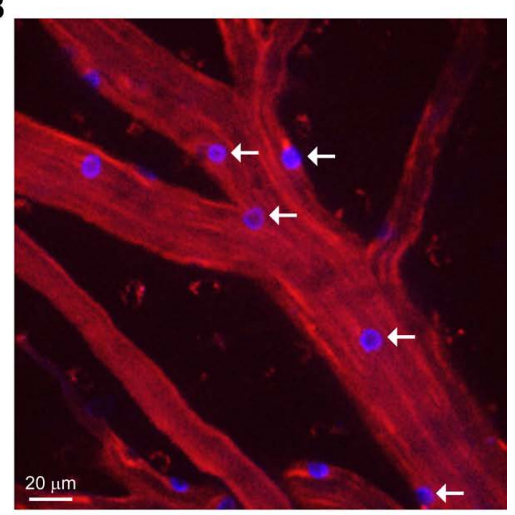

D

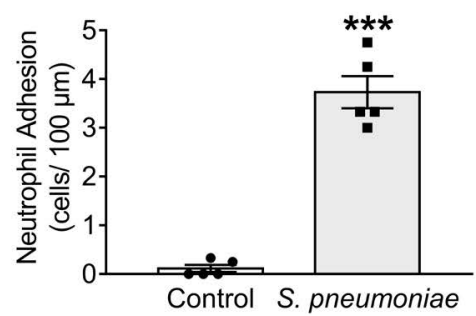

F

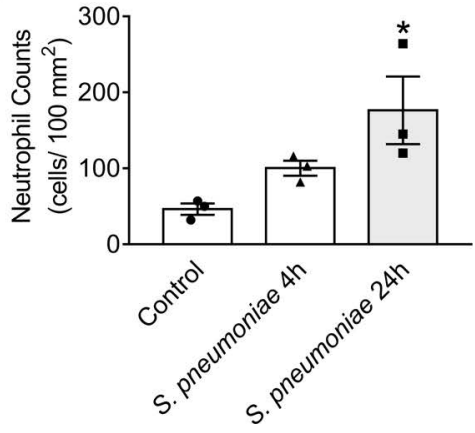

Figure 4. S. pneumoniae-infected mice have increased neutrophil recruitment into the brain. Mice were infected with S. pneumoniae for 24 hours. (A) Intravital microscopy image of the brain vasculature of control mice. Scale bar: $20 \mu \mathrm{m}$. (B) Intravital microscopy image of the brain vasculature in S. pneumoniae-infected mice at 24 hours after infection. Neutrophils are visualized in blue, labeled with anti-Ly6G mAb (clone 1A8). Endothelium is visualized in red, labeled with anti-CD31 mAb. Scale bar: $20 \mu \mathrm{m}$. (C) Quantification of neutrophil rolling flux $(n=5)$. (D) Quantification of neutrophil adhesion ( $n=5$ ). Data in $\mathbf{C}$ and $\mathbf{D}$ represent mean \pm SEM. ${ }^{* * *} P<0.001$ vs. control, unpaired 2 -tailed $t$ test. (E) Coronal brain section image showing adherent neutrophils in the inset (arrows showing neutrophils visualized in blue). Neutrophils were labeled with anti-Ly6G mAb (clone 1A8) and endothelium was labeled with anti-CD31 mAb. (F) Quantification of neutrophil adhesion in coronal sections at 4 hours (S. pneumoniae $4 \mathrm{~h}$ ) or 24 hours (S. pneumoniae $24 \mathrm{~h}$ ) after S. pneumoniae infection $(n=3)$. Data represent mean \pm SEM. ${ }^{*} P<0.05$ vs. control, 1 -way ANOVA followed by Dunnett's multiple comparisons test.

rolling and adhesion at 24 hours after infection (rolling $=0.57 \pm 0.16$ cells $/ \mathrm{min}$ and adhesion $=0.13 \pm$ 0.07 cells $/ 100 \mu \mathrm{m}$, mean $\pm \mathrm{SEM}, n=4, P<0.0001$ vs. $S$. pneumoniae infected). Unexpectedly, rolling and adhesion of neutrophils was also completely abolished (data not shown). Importantly, mice treated with anti-CCR2 $\mathrm{mAb}$ that had recovered from infection did not show memory impairment in the water maze test (Figure 7, E and F). These data suggest that the recruitment of early CCR2 ${ }^{+}$monocytes into the brain plays a crucial role in the cognitive impairment, and preventing their recruitment in the acute phase of sepsis seems to be a crucial step in order to protect the brain.

Wild-type but not CCR2 $2^{\text {def }}$ mice show microglial activation. Since an increase in vascular permeability did not affect the cognitive impairment, we focused on other potential alterations. Microglial activation plays a role in mediating inflammation associated with neurodegenerative illnesses (24). Using CX3CR $1^{\mathrm{GFP} / \mathrm{WT}}$ reporter mice in all experiments allowed for direct visualization of microglia, which are large cells with long dendrites probing the local environment (25). Microglial activation was assessed in wild-type fluorescent reporter (CX3CR1 $1^{\mathrm{GFP} / \mathrm{WT}} \mathrm{CCR} 2^{\mathrm{RFP} / \mathrm{WT}}$ ) mice by multiphoton microscopy. Nonactivated microglia have small irregular shaped soma and long extended processes, while activated microglia have an amoeboid-like appearance, with large and circular soma and short thick processes (26). Figure 8A shows the distribution of microglia in the brain of a control mouse. In control mice, microglia had a soma size of approximately $40.0 \mu^{2}$ (Figure 8, B and D), while in S. pneumoniae-infected mice, microglia had a mean soma size of $53.1 \mu \mathrm{m}^{2}$ (Figure 8, C and D), suggesting that microglia were activated. Microglia were also characterized by flow cytometry. Resting microglia were identified as CD $11 b^{+} \mathrm{CD} 45^{\text {lo }}$-expressing cells, whereas activat-

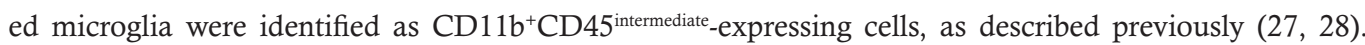
Microglia were activated at 24 hours after infection, as shown by a significant increase in the expression

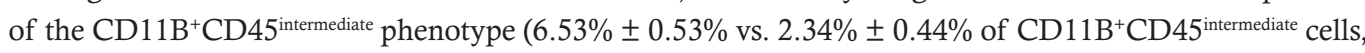


A

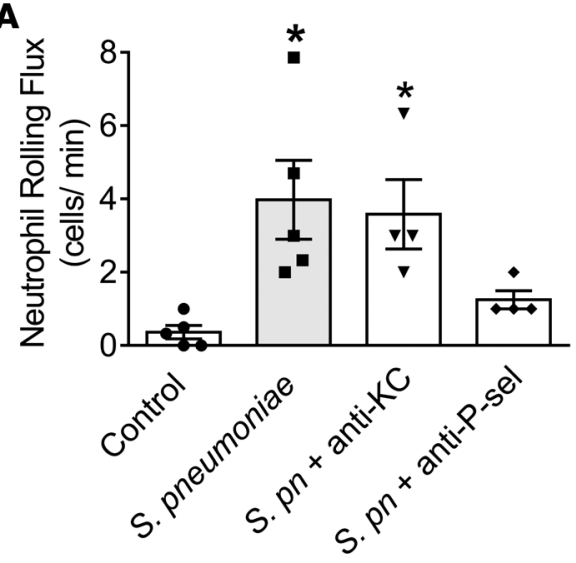

C

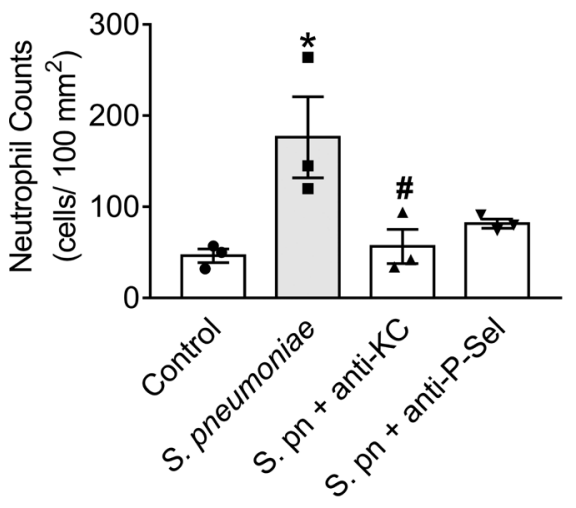

B

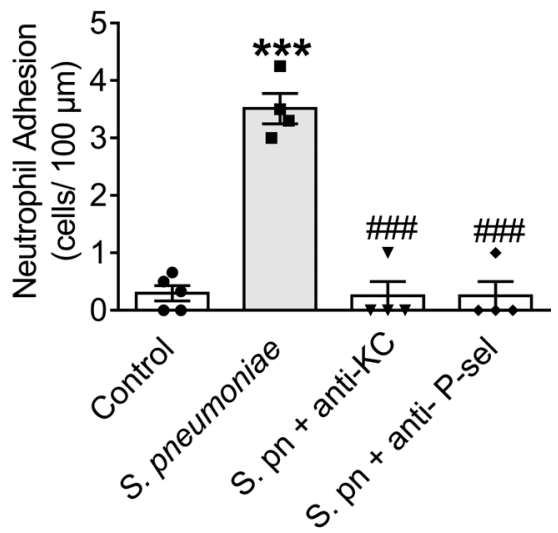

D

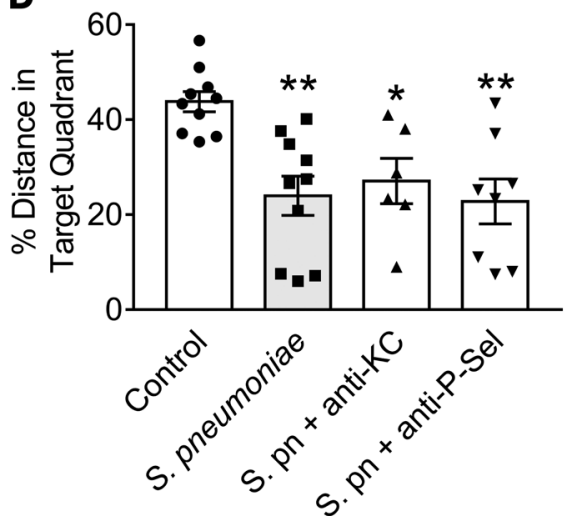

Figure 5. Inhibition of neutrophil recruitment into the brain does not have an effect cognitive impairment. Mice were infected with S. pneumoniae and immediately received anti-P selectin mAb (S. pn + anti-P-sel) or at 4 hours after infection received anti-KC $\mathrm{mAb}$ (S. pn + anti-KC). (A) Neutrophil rolling flux at 24 hours $(n=4-5)$. (B) Neutrophil adhesion at 24 hours after infection $(n=4-5)$. (C) Neutrophil adhesion in coronal brain section $(n=3)$. Data in $\mathbf{A}-\mathbf{C}$ represent mean $\pm \mathrm{SEM} .{ }^{*} P<0.05,{ }^{* * *} P$ $<0.001$ vs. control, ${ }^{\#} P<0.05$, \#\# $P<0.001$ vs. $S$ pneumoniae, 1-way ANOVA followed by Tukey's multiple comparisons test. (D) Morris water maze at 2 weeks after infection. Percentage of distance spent in the target quadrant on the fourth day after the platform was removed in control $(n=10)$, S. pneumoniae-infected $(n=$ 10 ), and S. pneumoniae-infected mice treated with anti-KC mAb $(n=6)$ or anti-P selectin ( $n=$ 8). Data represent mean $\pm \mathrm{SEM} .{ }^{*} P<0.05,{ }^{* *} P$ $<0.01$ vs. control, 1-way ANOVA followed by Dunnett's multiple comparisons test.

mean $\pm \mathrm{SEM}, n=6, P<0.05, S$. pneumoniae vs. controls, Figure 8E). Interestingly, $S$. pneumoniae-infected $\mathrm{CCR}^{\text {def }}$ mice showed no activation of microglia with a mean soma area of $40.3 \pm 1.03 \mu \mathrm{m}^{2}(\mathrm{mean} \pm \mathrm{SEM}$, $n=3$ ). As a whole, these data suggest that CCR $2^{+}$monocyte recruitment caused microglial activation across the blood-brain barrier independent of neutrophils or the increase in vascular permeability in $S$. pneumoniae pneumonia-induced sepsis.

\section{Discussion}

It is well appreciated that patients are in a state of hyperinflammation during the first few days of sepsis, clinically manifested by fever and tachycardia that are often accompanied by vasodilation, in which case fluid is required for any accompanying hypotension. In addition, respiratory support for lungs and dialysis for failing kidneys is often needed. Another very common sequelae of sepsis is delirium or SAE for which a specific pathogenesis has not been demonstrated, but some hypothesize may be due to inflammation of the brain (29). However, because this does not necessarily present an immediate life-threatening situation and is of unknown etiology, there is no interventional support provided for this organ dysfunction. Sedation is a standard of care, but this treats the symptom rather than the underlying cause. As such, over the last few years, a connection has been made among inflammation, SAE, and long-term cognitive impairment; however, directed therapy remains unavailable (29). Nevertheless, patients who recover from sepsis show various anatomical anomalies, including damage to the median temporal lobe, leading to impaired interregional communication, alterations in white matter (30), and neuronal death in parahippocampus and frontal regions (29). Consistent with these anatomical changes are reports that sepsis survivors exhibit impairments in memory and, to some extent, executive function a year or more after recovery from sepsis. These data show that events during the septic episode correlate with the long-term cognitive dysfunction.

In this study, we hypothesized that delirium or SAE in septic patients was the result of inflammation of the brain, leading to long-term cognitive impairment. Indeed, our cohort of patients that experienced delirium demonstrated significant underperformance in pattern and spatial recognition memory and delayed matching to sample behavioral tests. Numerous cytokines and chemokines were elevated in these patients, 
A

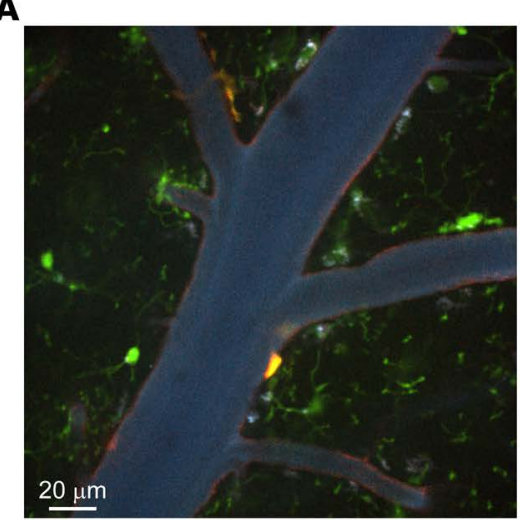

C

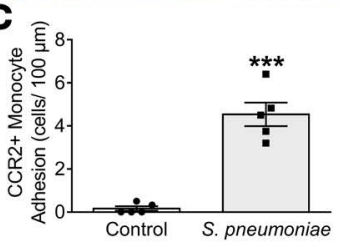

D

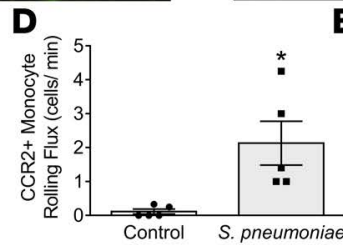

B

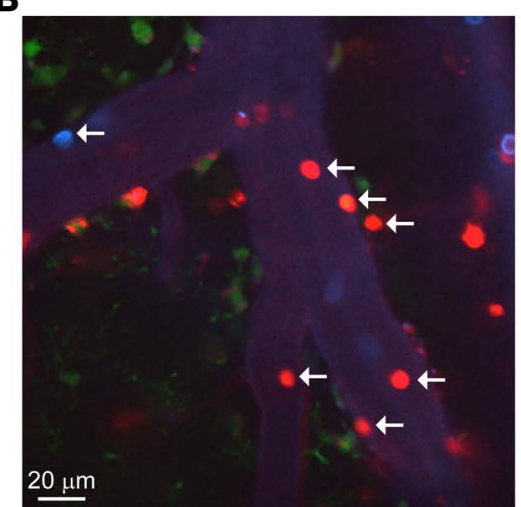

E

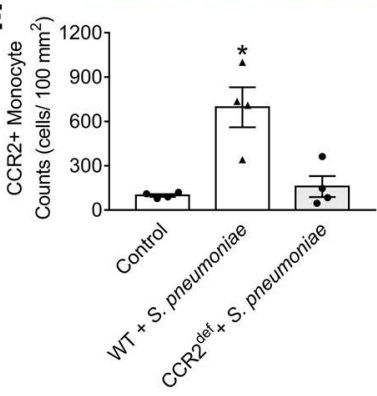

$\mathbf{F}$

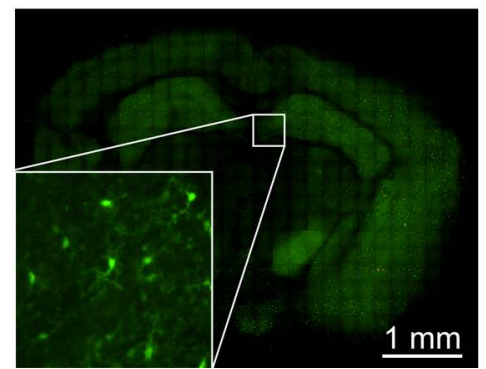

G

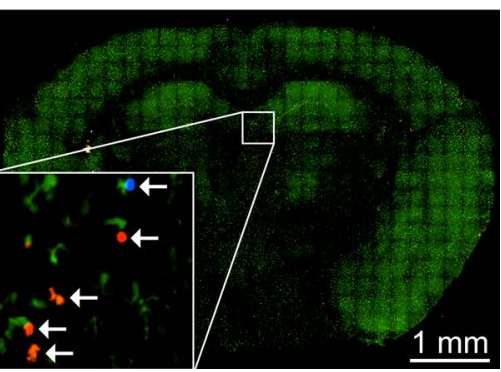

Figure 6. 5. pneumoniae infection increases CCR2 monocyte recruitment. Wild-type reporter mice CX3CR1 ${ }^{\text {CFP/WT } C C R 2 ~}{ }^{\text {RFP/WT }}$ were infected with S. pneumoniae. (A) Intravital microscopy image of control mouse brain vasculature. Scale bar: $20 \mu \mathrm{m}$. (B) Intravital microscopy image of $S$. pneumoniae-infected brain vasculature at 24 hours. Scale bar: $20 \mu \mathrm{m}$. (c) Quantification of CCR2 $2^{+}$monocyte rolling flux at 24 hours. (D) Quantification of CCR2 ${ }^{+}$monocyte adhesion at 24 hours. Data in $\mathbf{C}$ and $\mathbf{D}$ represent mean \pm SEM of $n=5$. ${ }^{*} P<0.05$, ${ }^{* * *} P<0.001$ vs. control, unpaired 2 -tailed $t$ test. (E) Quantification of monocyte adhesion in the coronal brain section at 4 and 24 hours after infection. Data represent mean \pm SEM of $n=3$. ${ }^{*} P<0.05$ vs. control, 1-way ANOVA followed by Dunnett's multiple comparisons test. (F) Coronal brain section image by confocal microscopy of a control mouse. Scale bar: $1 \mathrm{~mm}$. (G) Coronal brain section image by confocal microscopy of a S. pneumoniae-infected mouse at 24 hours after infection. Scale bar: $1 \mathrm{~mm}$. In the images neutrophils are visualized as blue, detected by anti-Ly6G mAb; CCR2 ${ }^{+}$monocytes are visualized as red (shown by arrows in $\mathbf{B}$ and $\mathbf{G}$ ), by the knockin fluorescent marker CCR2 ${ }^{\mathrm{RFP} / \mathrm{WT}}$; microglia are visualized as green, detected by the knockin fluorescent marker CX $3 \mathrm{CR}^{\mathrm{CFP} / \mathrm{WT}}$; and endothelium is visualized as red, by anti-CD31 mAb (in A and B).

consistent with the first part of our hypothesis. Interestingly, these responses were similar in all the studied patients infected with Gram-negative and/or Gram-positive bacteria and did not appear to be associated with a specific bacterial strain. To probe for causality, we used a previously established mouse model of $S$. pneumoniae pneumonia-induced sepsis (19) and identified pattern and spatial memory impairments using analogous behavioral tasks. Neither species had detectable signs of brain infection, and mice at 24 hours after infection showed similar increases in chemokines and cytokines, including KC, G-CSF, MCP-1, MIP- $1 \alpha$, and IP-10. In the mouse model, we found that neutrophils and CCR2 ${ }^{+}$inflammatory monocytes were both significantly recruited to the brain during the acute inflammatory phase of sepsis. However, unlike what we and others have observed in other infected organs and in other models of inflammation (31-33), neutrophils were by no means the predominate inflammatory cell recruited. Indeed, a much larger influx of inflammatory monocytes was noted, and when CCR2 ${ }^{+}$inflammatory monocyte recruitment was blocked, inflammation (microglial activation and neutrophil recruitment) was reduced and long-term signs of cognitive impairment were abrogated, consistent with the second part of our hypothesis. To our knowledge, our data are the first to show a role for $\mathrm{CCR} 2^{+}$inflammatory monocytes in long-term cognitive dysfunction following sepsis.

Our data would also suggest that neutrophils were not the inflammatory cells that were causative of long-term cognitive impairment in SAE. Even though we visualized significant neutrophil recruitment to the brain during $S$. pneumoniae pneumonia-induced infection, our data show that neutrophil recruitment was not associated with cognitive impairment. In fact, neutrophil recruitment may be secondary to CCR2 ${ }^{+}$ monocyte recruitment, as illustrated by our observation that monocyte recruitment in infected CCR2 $2^{\text {de }}$ mice was essentially abolished and this led to a reduction in other hallmarks of inflammation, including neutrophil recruitment and microglial activation. CCR2 is not found on neutrophils or microglia, suggesting that it is first and foremost the monocyte that is affected in CCR2 $2^{-/}$mice (34). Indeed, we observed a 
A
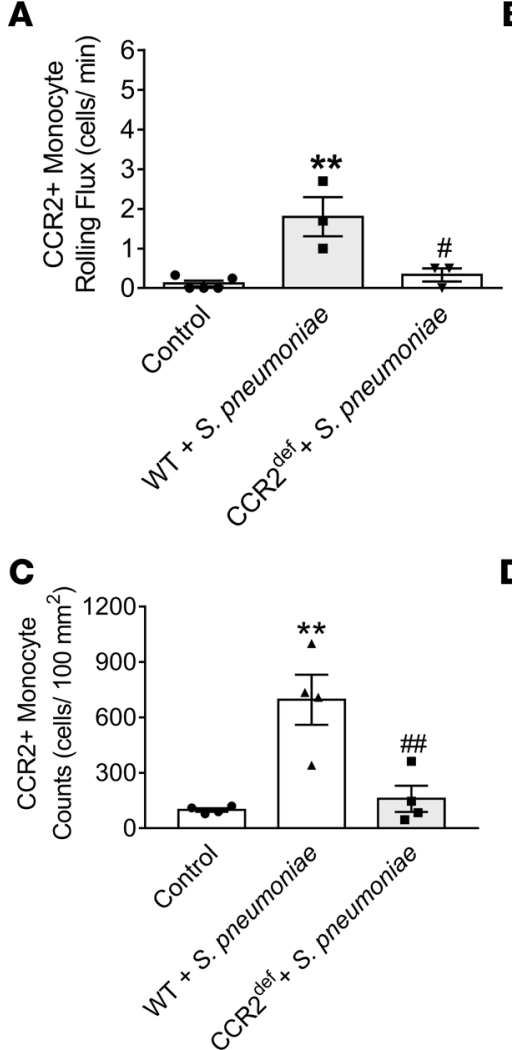

$\mathbf{E}$

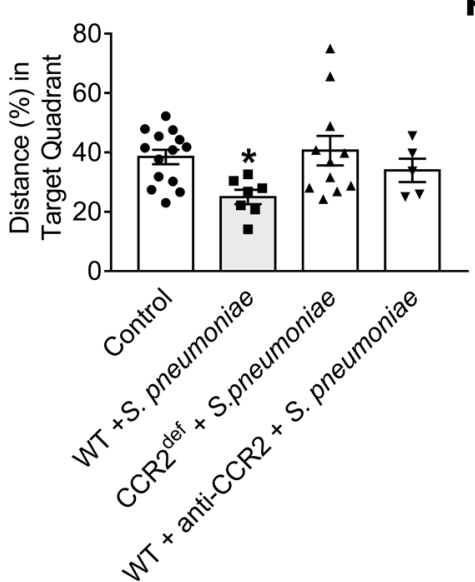

B

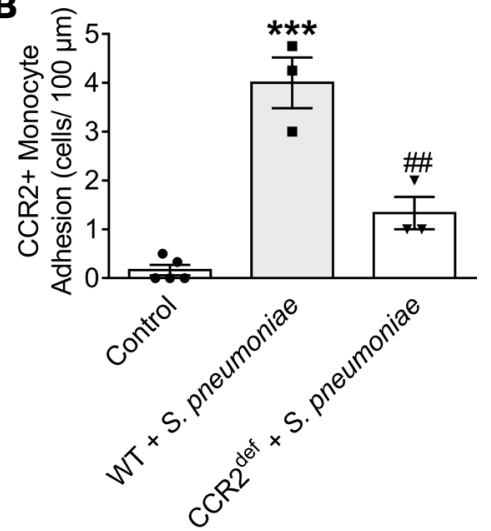

D

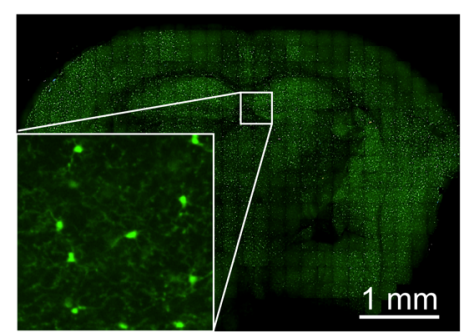

$\mathbf{F}$

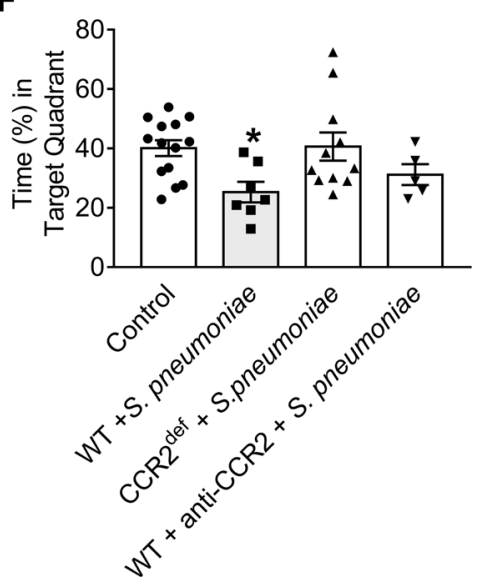

Figure 7. Effects of S. pneumoniaeinfection on CCR2 ${ }^{+}$monocyte recruitment and Morris water maze in CCR2 ${ }^{\text {def }}$ mice. Wild-type

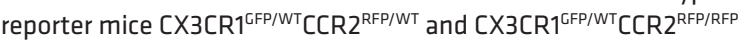
(CCR2 ${ }^{\mathrm{def}}$ ) mice were infected with S. pneumoniae for 24 hours. (A) Quantification of CCR2+ monocyte rolling flux. (B) Quantification of CCR2 ${ }^{+}$monocyte adhesion. (C) Quantification of CCR2 $2^{+}$monocyte counts in the coronal brain section by confocal microscopy. Data in A-C represent mean \pm SEM of $n=3-5 .{ }^{* *} P<0.01$, ${ }^{* *} P$ $<0.001$ vs. control, ${ }^{\# P}<0.05$ vs. WT + S. pneumoniae, ${ }^{\#} P<$ 0.01 vs. WT + S. pneumoniae, 1-way ANOVA followed by Tukey's multiple comparisons test. (D) Coronal brain image of CCR2 $2^{\text {def }}$ mice infected with S. pneumoniae for 24 hours. (E) Morris water maze and percentage of distance spent in the target quadrant on the fourth day. (F) Morris water maze and percentage of time in target quadrant. Wild-type mice, CCR $2^{\text {def }}$ mice, and mice treated with anti-CCR2 mAb (see Methods) were infected with S. pneumoniae and allowed to recover for 2 weeks, and at this time, the Morris water maze test was assessed. Data represent mean \pm SEM of $n=14$ in control, $n=7$ in WT + S. pneumoniae, $n=11$ in CCR2 $2^{\mathrm{def}}+$ S. pneumoniae, and $n=5$ in WT + anti-CCR2 + S. pneumoniae mice. ${ }^{*} P<0.05$ vs. control mice, 1 -way ANOVA followed by Dunnett's multiple comparisons test.

complete reduction in monocyte adhesion to the cerebrovasculature in CCR2 ${ }^{\text {def }}$ mice and complete abolition of cognitive impairment. This is almost certainly related to the fact that CCR2 ${ }^{\text {def }}$ mice have almost no circulating monocytes. Notably, treatment of wild-type mice with anti-CCR $2 \mathrm{mAb}$ completely abrogated $\mathrm{CCR}^{+}$monocyte recruitment into the brain at 24 hours and protected the brain from cognitive impairment, suggesting that the early wave of $\mathrm{CCR} 2^{+}$monocytes into the brain plays a crucial role in cognitive impairment. Interestingly, a recent study reported no impairment in monocyte recruitment into the brain in a model of cecal ligation and puncture in CCR2 $2^{-/-}$mice (35). This observation is difficult to explain in light of the fact that these mice have so few circulating monocytes, but perhaps the very long-term (up to 50 days) neuroinflammation may account for some small accumulation of monocytes to the continuously inflamed brain. In our model, there was a very obvious reduction in cerebrovascular brain inflammation, including monocyte adhesion at 72 hours and complete recovery by 2 weeks. It is worth highlighting that, while in an acute septic episode, monocytes may be the primary driver of delirium and long-term cognitive impairment, in other forms of sepsis with an ongoing neuroinflammation, the drivers of neuroinflammation and cognitive impairment may be multifactorial and more complex. 
A

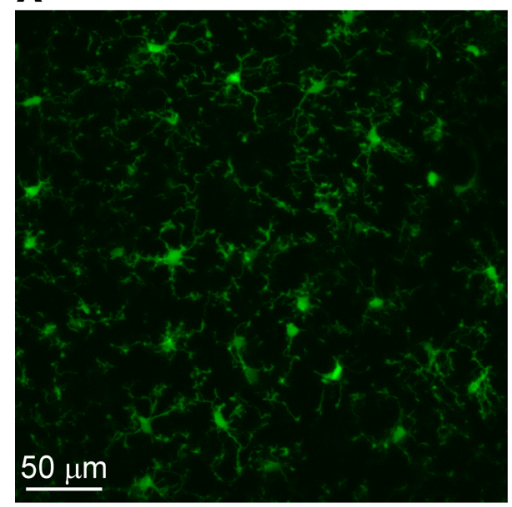

D

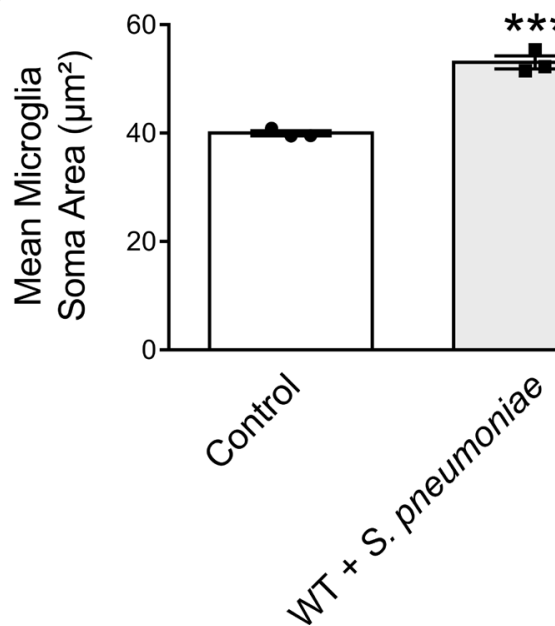

B

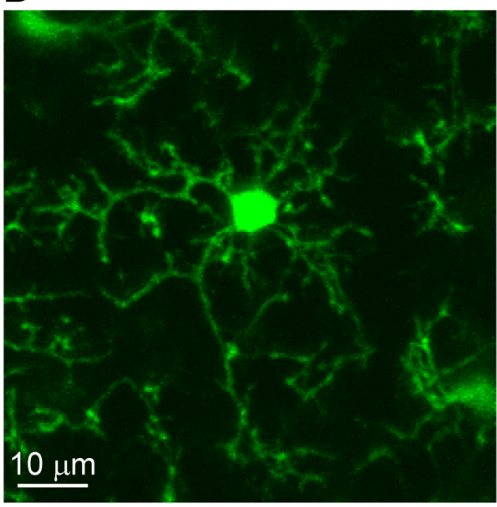

C

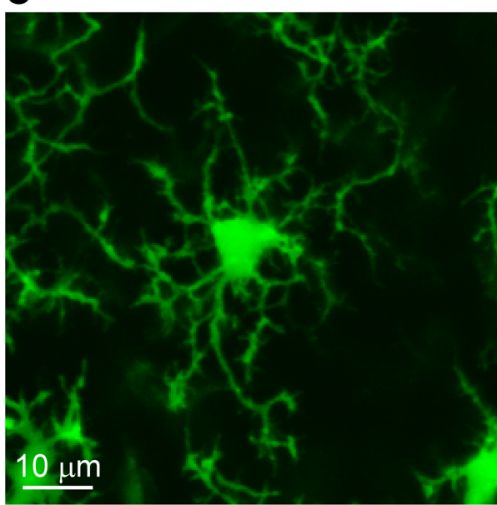

E

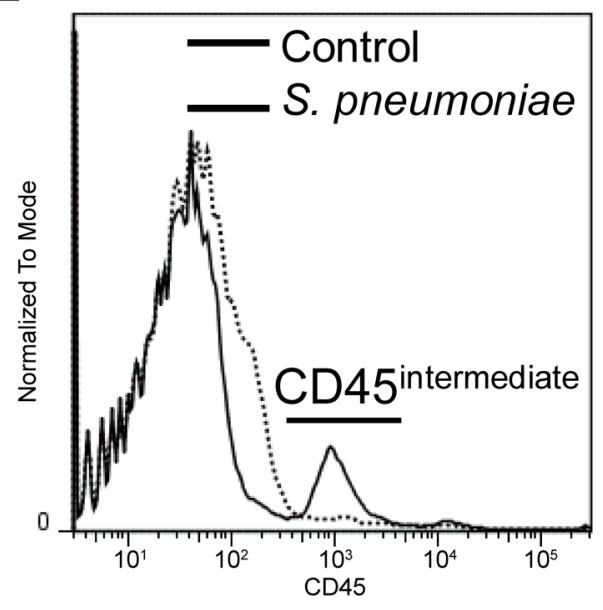

Figure 8. Effects of S. pneumoniae infection on microglia activation. Wild-type reporter mice CX $3 C R 1^{\mathrm{CFP} / W T} C C R 2^{\mathrm{RFP} / W T}$ and $C X 3 C R 1^{\mathrm{GFP} / W T} C C R 2^{\mathrm{RFP} / \mathrm{RFP}}$ (CCR2 ${ }^{\text {def }}$ ) mice were infected with S. pneumoniae for 24 hours. (A) Image of microglia Z-stacks, shown as the maximum intensity projection of 20 - $\mu$ m stack obtained by multiphoton microscopy of a S. pneumoniae-infected CX3CR1 ${ }^{\mathrm{CFP} / W T}$ CCR2 ${ }^{\mathrm{RFP} / \mathrm{WT}}$ mouse at 24 hours. Scale bar: 50 mm. (B) Inactivated microglia, characterized by a soma area of 0-50 $\mu \mathrm{m}^{2}$. Scale bar: $10 \mu \mathrm{m}$. (C) Image of activated microglia 24 hours after infection in a CX3CR ${ }^{\text {CFP/WT }}$ CR2 ${ }^{\text {RFP/WT }}$ mouse. Scale bar: $10 \mu \mathrm{m}$. (D) Quantification of microglia activation. Data represent mean \pm SEM of $n=3$. ${ }^{* *} P<0.001$ vs. control, unpaired 2-tailed $t$ test. (E) Microglia C11b+CD45intermediate expression by flow cytometry. Brain cells from control and 5 . pneumoniae-infected mice for 24 hours were isolated as previously described and labeled with CD11b and CD45. The CD11b+ cells were gated and analyzed for CD45 intermediate expression. The representative histogram $(n=3)$ shows the increase in CD45 intermediate expression in brain microglia from $S$. pneumoniae pneumonia-infected mice versus controls.

Interestingly, in the last 3 years, a significant $\mathrm{CCR} 2^{+}$monocyte recruitment to the brain has been reported in other neuroinflammatory diseases. Morganti et al. (36) and Hammond et al. (37) have reported a link to acute cognitive impairment and CCR2 $2^{+}$monocyte recruitment in traumatic brain injury and stroke, respectively, showing the importance of $\mathrm{CCR} 2^{+}$monocyte recruitment in different neurodegenerative disorders that have as their commonality acute inflammation. The specific mechanism of long-term cognitive impairment remained unknown. While neutrophils have been implicated through the production of large amounts of oxidants, capable of generating significant oxidative stress, monocytes lack this capacity. In addition, Varvel et al. (38) have shown that $\mathrm{CCR}^{+}$monocytes play a key role in the neuroinflammation and morbidity after status epilepticus, a condition associated with cognitive decline. Inflammatory monocytes have been reported to induce brain demyelination in experimental autoimmune encephalomyelitis (39), and we did see monocytes leave the vasculature and enter the parenchyma, potentially causing overt injury to neurons or other structures. However, in our experimental conditions, no morphological changes were observed in the hippocampus of mice recovered from sepsis. In our hands the CCR2 ${ }^{+}$monocytes caused impairment in memory, suggesting damage to the hippocampus. Damage to the hippocampus and associated structures can disrupt some forms of memory (40), particularly spatial memory (41-43). However, we could see no overt gross changes to the hippocampus anatomy, a common finding in more subtle behavioral changes. The functional deficit was limited and does not appear to significantly 
affect amygdala circuitry. This was indicated by the lack of change in context fear conditioning, a form of learning that involves amygdala connections $(44,45)$. In addition, the prolonged disturbance of the blood-brain barrier has been recognized as a potential contributor in the pathogenesis of neurological diseases, such as late-onset Alzheimer's disease, multiple sclerosis, etc. $(46,47)$. However, the acute increase in blood-brain permeability was not sufficient to explain the long-term cognitive impairment in our study.

In summary, this is the first demonstration to our knowledge that CCR2 $2^{+}$monocyte recruitment to the brain plays an important role in SAE-induced long-term cognitive impairment. Further, S. pneumoniae pneumonia-induced sepsis models many aspects of human SAE disease, which implies that the protective effects of preventing CCR2 ${ }^{+}$monocyte recruitment in mice may be a potential therapeutic target for human diseases. Our Gram-positive sepsis mouse model may extend to Gram-negative sepsis, as the human patients presented similar responses independent of the type of bacterial infection. It is worth noting that essentially all clinical trials in the sepsis arena focus on 28 day all-cause mortality and not long-term improvement to individual organs, including long-term cognitive health. The increase in survival rates in patients with sepsis brings with it the responsibility to ensure the highest possible quality of life after illness. This work represents the identification of a potential mechanism for preserving cognitive function following SAE.

\section{Methods}

\section{Study design}

The main goal of this study was to investigate the role of the activation of the innate immune system in SAE and cognitive impairment. Our hypothesis was that the inappropriate activation of the immune system and cerebral leukocyte recruitment leads to brain inflammation that contributes to the development of SAE and long-term cognitive impairments. To test our hypothesis, a mouse model of $S$. pneumoniae pneumonia-induced sepsis with multiple organ dysfunction, including SAE, was characterized and established. This mouse model modeled several aspects of the human disease, as shown in Results. The sample size in the human studies was determined by the patients we were able to recruit for a period of 4 years. In the human studies the investigators were not blind to the condition. With regard to the animal studies, the sample size was derived from our previous published studies $(13,19)$. All the initial mouse behavioral experiments characterizing the mouse model were assessed and analyzed by investigators that were blinded to the information about treatment groups. Once the mouse model was characterized and established the experiments were no longer blinded.

\section{Human studies}

Twenty subjects (eleven sepsis survivors) were recruited through a follow-up clinic for patients who had been admitted to the ICU for treatment of systemic inflammatory response syndrome 12-18 months prior to study participation. Inclusion criteria included a history free of known mental or psychological illness and no prior brain injury, such as trauma, stroke, or evidence of dementia or cognitive dysfunction. All participants were between the ages of 18 and 60 years. Participants in each condition were matched for sex, age, and educational achievement.

\section{Cambridge Neuropsychological Automated Test Battery}

Subjects performed the Cambridge Neuropsychological Automated Test Battery under the supervision of trained neuroscientists. Subjects completed a battery of tests that includes a motor screen, pattern recognition memory, spatial recognition memory, delayed matching to sample, paired associates learning, spatial span, spatial working memory, stockings of Cambridge, big/little circle, and intradimensional/extradimensional shifting. Experimenters aborted tasks if the task was too difficult for participants to solve. The variation in the degrees of freedom reported in the results reflect the sepsis survivors for whom performance on those tasks had been aborted.

\section{Mice}

Wild-type C57BL/6 and CXCR2 $2^{-/-}$male mice were originally purchased from The Jackson Laborato-

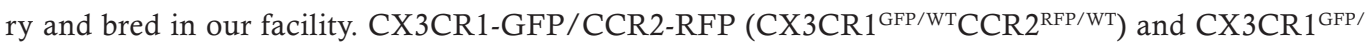
${ }^{\text {WT } C C R} 2^{\text {RFP/RFP }}\left(C C R 2^{\text {def }}\right.$ ) reporter mice on C57BL/ 6 background were donated by Richard Ransohoff (Cleveland Clinic, Cleveland, Ohio, USA). Male mice aged between 6 and 10 weeks were used for most experiments unless specified otherwise. 
Antibody treatment to inhibit neutrophil recruitment

Anti-KC mAb (25 $\mu$ g, MAB453, R\&D Systems) was administered at 4 hours of $S$. pneumoniae infection. Anti-P selectin mAb (75 $\mu \mathrm{g}$, RB40.34, BD Biosciences) was administered immediately after $S$. pneumoniae infection.

Antibody treatment to inhibit CCR2 ${ }^{+}$monocyte recruitment

Anti-CCR2 mAb (40 $\mu \mathrm{g}, \mathrm{MC}-21)$ was administered just before infection. The antibody was provided by Matthias Mack, Universitätsklinikum Regensburg, Innere Medizin II — Nephrologie).

\section{Mouse model of infection}

S. pneumoniae, SPN 49619 strain, a clinically pathogenic bacteria originally obtained from ATCC was grown as previously described (19). $50 \mu \mathrm{l}$ bacteria in PBS were directly injected into the lungs by transoral intratracheal injection. For control (sham) mice, the same volume of PBS was injected into the lungs by transoral intratracheal injection. The model was previously characterized and published (19) and caused profound lung inflammation and pneumonia; over time, it lead to multiple organ dysfunction. S. pneumoniae, harvested at $\log$ phase, were injected intratracheally in the mice at OD $600=0.4$. A dose of bacteria of $2 \times 10^{7}$ to $6 \times 10^{7} \mathrm{CFU}$ conferred approximately $30 \%$ mortality at 36 hours.

\section{MRI of mouse brains}

Imaging was performed by the Experimental Imaging Centre, Cumming School of Medicine, University of Calgary. The RARE T2W and FLASH multislice series of MRI brain images were saved as single slices and processed using the SPIN software. Quantification was performed by calculating the voxel-based volumetrics using AMIRA software. Individual images from the T2W series were saved as dicom images using SPIN, and these were used for the multislice analysis and volumetric analysis. The contrast of the image was adjusted so that the best white/gray distinction could be seen. In each of the images, the hippocampus was selected in the AMIRA software using the lasso and the brush tools. The ventricles and surrounding areas were not selected, and the volume of this area was measured using the material statistics tool in AMIRA. This process was repeated for all of the MRI brain images, and the data were analyzed using GraphPad software.

\section{Mouse brain preparation for intravital microscopy}

This technique was performed as previously described (48). Briefly, mice were anesthetized intraperitoneally with $200 \mathrm{mg} / \mathrm{kg}$ ketamine (Bayer Animal Health) and $10 \mathrm{mg} / \mathrm{kg}$ xylazine (Bimeda-MTC), and the jugular vein was cannulated to administer labeled antibodies and/or additional anesthetic as required. Mice were placed in a prone position on a brain board. Body temperature was maintained at $37^{\circ} \mathrm{C}$ using an infrared heat lamp. The brain was prepared for in vivo microscopic observation. A craniotomy was performed using a high-speed drill, and the dura mater was removed to expose the brain vasculature. The mouse brain remained fully perfused and was placed on an upright spinning disk confocal microscope. Neutrophils in the brain microvasculature were visualized with anti-mouse Ly6G mAb conjugated to APC (clone 1A8, BioLegend), and the vascular endothelium was visualized using anti-mouse CD31 mAb conjugated to PE (clone 390, eBiosciences). The antibodies were injected intravenously and immediately bound to the target protein causing the desired cell-type to be fluorescent. CCR $2^{+}$inflammatory monocytes and CX3CR $1^{+}$ patrolling monocytes were visualized in CX $3 \mathrm{CR} 1{ }^{\mathrm{GFP} / \mathrm{WT}} \mathrm{CCR} 2^{\mathrm{RFP} / \mathrm{WT}}$ knockin mice.

\section{Upright spinning disc confocal microscopy}

The mouse brain intravital microscopy was visualized using an Olympus BX51WI upright microscope (Olympus) equipped with a confocal light path (Wave-Fx, Quorum) based on a modified Yokogawa CSU-X1 head (Yokogawa Electric Corporation) with a $20 \times / 0.95 \mathrm{~W}$ (LUMPLANFL) water immersion objective. Up to 3 laser excitation wavelengths (491, 561, and $640 \mathrm{~nm}$; Cobalt) were used in rapid succession and visualized with the appropriate band-pass filters (Semrock). A back-thinned EM-CCD 512 $\times 512$ pixel camera (C9100-13, Hamamatsu) was used for fluorescence detection. Volocity acquisition software (PerkinElmer) was used to drive the microscope. All images were recorded and saved for postacquisition analysis using the Volocity program. Two to five vessels were recorded in each mouse, and the results were averaged. The number of rolling and adherent neutrophils and monocytes was determined offline during video playback analysis. Adherent neutrophils and monocytes were defined as the cells that remained stationary for more than 30 seconds. 
Mouse brain slice preparation for stitched images of brain and hippocampus

Mice were anesthetized intraperitoneally with $200 \mathrm{mg} / \mathrm{kg}$ ketamine (Bayer Animal Health) and $10 \mathrm{mg} /$ $\mathrm{kg}$ xylazine (Bimeda-MTC). The jugular vein was cannulated in order to administer labeling antibodies. The antibodies were allowed to circulate for 15 minutes. Then, mice were perfused with $25 \mathrm{ml}$ of $1 \times$ PBS through the right ventricle of the heart. The brain was removed, placed on ice for 10 minutes, and sliced into 2-mm thick coronal sections using a brain matrix. The coronal brain section containing the hippocampus was mounted onto slides to be visualized with an inverted spinning disc confocal microscope.

Coronal brain sections were visualized using an Olympus IX81 inverted microscope (Olympus) equipped with a confocal light path (Wave-Fx, Quorum) based on a modified Yokogawa CSU-X1 head (Yokogawa Electric Corporation) with a $4 \times / 0.16$ (UPLANSAPO) and 10×/0.40 (UPLANSAPO) air objectives. Excitation lasers at 491, 561, and $635 \mathrm{~nm}$ to excite GFP, PE/RFP, and APC were used, respectively, and visualized with the appropriate band-pass filters (Semrock). A back-thinned EM-CCD $512 \times 512$ pixel camera (C9100-13, Hamamatsu) was used for fluorescence detection. Volocity acquisition software (PerkinElmer) was used to drive the microscope. Neutrophils in the brain were visualized with anti-mouse Ly6G mAb conjugated to APC (clone 1A8, BioLegend). Endothelial cells were visualized with anti-CD31 mAb conjugated

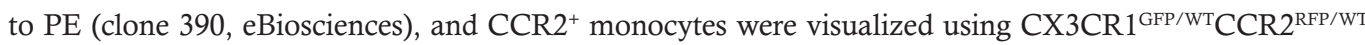
knockin mice. All images were recorded and saved for postacquisition analysis using the Volocity program.

Volocity Acquisition software was used to stitch the images together for the brain coronal section. Intensity of the neutrophil (visualized in blue) and monocytes (visualized in red) was analyzed so that differences in background fluorescence between experiments could be accounted for and to eliminate background autofluorescence. Contrast was adjusted to minimize autofluorescent background staining, and a minimum brightness threshold was set to yield only positive staining. The same contrast and threshold values were applied to all images from all treatment groups within the experiment. Cells were counted using the "Measurements Option," and the minimum cell size was set up at $40 \mu \mathrm{m}^{2}$ and the maximum cell size was set up at $200 \mu \mathrm{m}^{2}$. These cell sizes were chosen, as the average size of neutrophils is $7-10 \mu \mathrm{m}$ in diameter (49), and the average size for monocytes can vary from 8-14 $\mu \mathrm{m}$ in diameter (50). The number of cells was given under "Summary Statistics," and the fluorescence seen outside of the brain was subtracted from the counted cells to give the total number of cells in the stitched brain area. The brain area was measured and the number of cells stated by $100 \mathrm{~mm}^{2}$.

\section{Multiphoton intravital microscopy to visualize microglial activation}

The intravital brain preparation was visualized using a multiphoton microscope (Olympus BX61W1 FV300 modified, Olympus) using a $\times 20 / 1.0$ XLUMPlanFl water immersion objective equipped with a modified FV300 unit and Ti:sapphire laser. The laser emitted red and near-infrared light in the range of 680-1,020 $\mathrm{nm}$. A dichroic filter cube placed before the photomultiplier tube detectors allowed for simultaneous 2-color imaging. The filter cube was assembled from a beamsplitter and 2-photon detector emission filters (RXD2 [GFP] and RXD4 [RFP], Olympus). Olympus Fluoview 5.0 software was used to drive the microscope. GFP fluorescently labeled microglia and RFP fluorescently labeled CCR2 monocytes were visualized in the $\mathrm{CX} 3 \mathrm{CR} 1^{\mathrm{GFP} / \mathrm{WT}} \mathrm{CCR} 2^{\mathrm{RFP} / \mathrm{WT}}$ knockin reporter mice, and $Z$-stack images were acquired at 3 points of view per mouse (surface to $240 \mu \mathrm{m}$ in depth, $1 \mu \mathrm{m}$ intervals).

\section{Analysis of microglial images obtained from the multiphoton microscope}

Microglia images were analyzed by ImageJ software (National Institutes of Health) as previously described (26). Microglial Z-stacks were shown as maximum intensity projection of $20-\mu \mathrm{m}$ stacks. Then, the microglial cell perimeter outline was traced to threshold and eliminated background fluorescence. Microglial soma was traced and the area was measured. Microglia were considered activated when the soma were greater than $50 \mu \mathrm{m}^{2}(26)$.

\section{Assessment of microglia activation by flow cytometry}

Brain cells were isolated using a previously described protocol $(51,52)$. The samples were analyzed on a BD FACSCanto flow cytometer using FACSDiva software and FlowJo software (BD Biosciences). The expression of CD11b coupled with the differential expression of CD45 antigen was used to distinguish between resting/activated microglia and cerebral infiltrating monocytes. Resting and activated microglia were defined as $\mathrm{CD} 45^{10} \mathrm{CD} 11 \mathrm{~b}^{+}$and $\mathrm{CD} 45^{\text {intermediate }} \mathrm{CD} 11 \mathrm{~b}^{+}$cells, respectively; infiltrating monocytes were defined as CD45 ${ }^{\mathrm{hi}} \mathrm{CD} 11 \mathrm{~b}^{+}$cells (27). 


\section{Blood-brain barrier permeability assay}

Permeability across the blood-brain barrier was studied using a noninvasive whole body imaging InVivoXtreme 4MP (BRUKER, formerly Carestream). Brain permeability was assessed ex situ 24 hours after treatment in either control $S$. pneumoniae-infected or pertussis toxin-treated animals ( $200 \mathrm{ng} / \mathrm{d}$ from B. pertussis for 2 consecutive days treated as a positive control [\#180 Islet activating protein, List Biological Laboratories]). To visualize permeability changes, animals were injected i.v. 30 minutes prior to imaging with 200 $\mu 1$ of a 1\% Evans Direct Blue solution in PBS (Direct Blue 53, Sigma-Aldrich). To detect the Evans Direct Blue in the tissue $650 \mathrm{~nm}$ excitation and $700 \mathrm{~nm}$ emission wavelength filters were used.

The imaging protocol for each time point contained two steps: (a) reflectance imaging (2-second exposure time) and (b) fluorescent imaging at 650/700 (5-second exposure time). Images where quantified using Bruker molecular imaging software (version 7.1.3.20550) using a constant region of interest for all animals, which outlined the ventral and dorsal anatomical outline of the brain.

\section{Quantitation of cytokines}

Human blood was extracted at 24 hours after admission to the Foothills Medical Centre to measure 48 cytokines, including IL-6; IL-8; TNF- $\alpha$; TNF- $\beta$; IL-1 $\beta$; MCP-1; G-CSF; GM-CSF; IFN- $\gamma$; IFN- $\alpha 2$; IL-10; IL-12 (p40); IL-12 (p70); IL-15; IL-16; IL-17; IL-18; IL-2; IL-3; IL-4; IL-5; IL-7; MIP-1 $\alpha$; MIP-1 $\beta$; IL-13; IL-9; IP-10; RANTES; IL-1 $\alpha$; IL-1 $\beta$; IL-1Ra; IL-2R $\alpha$; CTACK; GRO- $\alpha$; HGF; LIF; MCP-3; M-CSF; MIF; MIG; $\beta$-NGF; SCF; SCGF- $\beta$; SDF-1 $\alpha$; TRAIL; bFGF; eotaxin; PDGF-bb; and VEGF by Luminex.

Mouse blood and cerebrospinal fluid (CSF) from the cistern magna, obtained as previously described by Liu et al. (53), were collected to measure the levels of 25 cytokines, including IL-6; KC; TNF- $\alpha$; IL-1 ; MCP-1; G-CSF; GM-CSF; IFN- $\gamma$; IL-10; IL-12 (p40); IL-12 (p70); IL-15; IL-17; IL-2; IL-4; IL-5; IL-7; MIP-1 $\alpha$; MIP-1 $\beta$; MIP-2; IL-13; IL-9; IP-10; and RANTES by Luminex. The assay was performed according to the manufacturer's instructions (EMD Millipore Corporation, Merck KGaA).

\section{Behavior assessment in mice}

Several tests were used to assess behavior in mice. All the behavioral tests were conducted at least at 14 days after $S$. pneumoniae infection.

Open field. The open field test was carried out in a square container $(90 \mathrm{~cm} \times 90 \mathrm{~cm} \times 40 \mathrm{~cm})$. Naive mice were placed individually in the center of the open field and were allowed to freely explore for $10 \mathrm{~min}$ utes. The open field was divided into 3 regions: center, middle, and outer. The movements of the mice were tracked using an overhead camera and analyzed with SMART video tracking system (PanLab). The total distance traveled, speed, and percentage of the total distance traveled that was spent in each region of the open field was recorded (54).

Y-maze. The Y-maze was constructed out of 3 Plexiglas arms of equal size joined together in a Y configuration and mounted on a piece of plywood. Each arm is $40-\mathrm{cm}$ long and 10-cm wide with 12-cm high walls. The floor of each arm was white and the walls were clear. The apparatus was placed at ground level, and all sessions were video captured for subsequent analysis. Individually, the mice were allowed to explore 2 of the 3 arms for a total of 10 minutes. The third arm was blocked with a piece of removable Plexiglas. After a 30-minute rest period, mice were individually returned to the maze for 5 minutes with all arms open. The number of entries, time spent, and percentage of the total distance traveled in each arm were quantified (55).

Elevated plus maze. The elevated plus maze consisted of 4 black Plexiglas arms, 2 open arms $(67 \mathrm{~cm} \times$ $7 \mathrm{~cm})$, and 2 enclosed arms $(67 \mathrm{~cm} \times 7 \mathrm{~cm} \times 17 \mathrm{~cm})$ that form a cross shape with the 2 open arms opposite each other. The maze was elevated $55 \mathrm{~cm}$ above the floor. Animals were placed in the center of the maze and allowed to explore for 10 minutes. A camera was mounted directly above the maze to record the behavior of the animal for subsequent analysis. The numbers of entries into as well as the time spent in the open versus enclosed arms were recorded. An anxiety ratio was calculated for each animal by dividing the amount of time spent in the open arms by the total time in the open plus enclosed arms.

Morris water maze. The mouse was placed in a pool of water with spatial cues around the room to learn the location of a submerged hidden platform. Acquisition curves and how quickly the mouse learned the hidden platform location were used to assess spatial learning. Memory for the target location (i.e., spatial memory) was assessed via a probe trial wherein the platform was removed and the time spent in the platform location was recorded. 
The water maze consisted of a circular swimming pool $1.13 \mathrm{~m}$ in diameter and $0.5 \mathrm{~m}$ in height constructed of white fiberglass. The pool is located in a room with various spatial cues. The pool was filled with $24.5^{\circ} \mathrm{C}$ water $\left( \pm 0.5^{\circ} \mathrm{C}\right)$ rendered opaque with skim milk powder. An escape platform was submerged $1 \mathrm{~cm}$ below the surface of the water. On the first day, mice were given morning and afternoon training sessions separated by 4 hours. Each session consisted of 4 trials, where mice were released from the north, east, south, and west points of the pool, with the platform in the center of an arbitrarily defined quadrant that was held constant. Between each release point, mice were placed back in their home cage for a 10-minute intertrial period. In each of the trials, mice were given 60 seconds to locate the platform. Mice remained on the platform for 10 seconds until the experimenter removed them to a holding cage. Mice unable to find the platform within the 60 seconds were placed on the platform for 10 seconds, thus ending the trial. After 3 days of training, a probe trial was conducted. The platform was absent during the probe trial, and the mice swam for the total 60 -second duration before being removed from the pool. Following the probe test, mice were given 4 trials to learn the location of a visible platform (a dark sponge is attached to the top of the platform so the mice were able to find it easily), thereby eliminating the possibility of sensory, motor, or motivational impairments driving observed impairments. Mice were recorded by a video camera mounted directly above the pool and connected to a computer with a video tracking system using SMART software (PanLab), and the data were processed using Microsoft Excel. This allowed for calculation of latency to find the platform during the 3 days of training as well as percentage distance and time spent in the platform-containing quadrant.

Immediate shock deficit. The immediate shock deficit test was conducted over 3 days based on the methods outlined in Fanselow et al. (22). On day 1, mice were placed in either context A (square chamber with black walls and a grid floor [Kinder Scientific]) or context B (rectangular blue plastic chamber with bedding) for 10 minutes. Mice were video recorded for the duration of the experiment. The amount of time spent freezing was quantified and defined by the complete absence of movement. On day 2, mice were placed into context $\mathrm{A}$ and immediately given a foot shock ( 1 second, $0.75 \mathrm{~mA}$ ). Mice were removed from the context 30 seconds after the shock. On day 3, mice were returned to context A for 8 minutes and scored for freezing (lack of movement), no shocks were administered. Each minute within the entire session was scored for freezing.

Cued and contextual fear conditioning. Fear conditioning was conducted based on methods outlined in Saxe et al. (23) with slight modifications. Briefly, fear conditioning was conducted in a chamber with plastic insertable walls and a metal rung floor $3 \mathrm{~cm}$ above the base of the apparatus (Kinder Scientific). The internal dimensions of the chamber were approximately $20 \mathrm{~cm} \times 15 \mathrm{~cm} \times 25 \mathrm{~cm}$. The behavior of the mice was recorded by a digital video camera directly mounted above the conditioning chamber. The amount of time spent freezing was quantified and defined by the complete absence of motion.

Fear conditioning was conducted over 3 days. On day 1, mice were placed in the conditioning chamber with white walls and receive 3 pairings between a tone (20 seconds, $80 \mathrm{~dB})$ and a coterminating shock (1 seconds, $0.5 \mathrm{~mA}$ ). The intertrial interval between each of the pairings was 2 minutes. Mice were first placed in the chamber and habituated to the surroundings for 2 minutes, after which the first tone shock pairing was presented. The chamber was cleaned with $70 \%$ ethanol after each mouse. On the second day, the procedure and context were changed in several ways to test conditioned fear of the tone in the absence of contextual cues associated with the shock. The chamber consisted of a striped cylinder with a plastic floor; the chamber was scented by placing a vanilla-scented paper towel at the base of the apparatus; chambers were cleaned with a nonalcohol disinfectant; and mice were kept in a different room before testing. Each mouse was placed in the chamber for approximately 6 minutes. A tone was presented twice for 20 seconds, 120 seconds, and 260 seconds after a mouse was placed into the chamber. No shocks were administered. Freezing was scored 1 minute before the first presented tone (pretone) and during the 20 second of the first tone (tone). On day 3, mice were tested for conditioned fear in the original training context. The testing procedure and context were identical to those used on day 1, except that the shock was not presented. Each minute within the entire session was scored for freezing.

\section{Statistics}

Human behavioral data were analyzed with SPSS Statistics v21 for Mac. All the other data were analyzed using GraphPad Prism 6 (GraphPad Software Inc., version 7.0). Values are expressed as mean \pm SEM. We assessed the statistical significance of the difference between two sets of data using an unpaired, 2-tailed $t$ test. 
To analyze the difference between more than 2 sets of data a 1-way ANOVA followed by a multiple comparisons follow-up test was used. To analyze the difference in the cytokine expression in the human samples Mann-Whitney $U$ test was used. All comparisons were 2-tailed, and $P<0.05$ was considered to be significant. The numbers of samples used is indicated in the figure legends.

\section{Study approval}

All human procedures were approved by both University of Calgary and University of Lethbridge institutional ethics committees under protocol 2011-089. All mouse procedures were approved by the University of Calgary Animal Resource Committee under protocol AC12-0205 and AC16-0211 and followed the Canadian Council on Animal Care guidelines.

\section{Author contributions}

GA conducted, designed, and supervised animal experiments; analyzed data; wrote and edited the manuscript. ELZ collected human data, analyzed data, and revised the manuscript. CLS conducted and analyzed in vivo and in vitro experiments and wrote manuscript. DK recruited and coordinated human data. LAC, BWW, SCS, BP, RN, and NJ conducted in vivo experiments. CNJ and MMK conducted in vitro experiments. JCS collected and analyzed human data. CJD assessed and recruited human patients, planned the project, and edited the manuscript. RJS designed, analyzed, interpreted human research and wrote and edited the manuscript. PK planned and supervised the entire study with CJD and RJS and wrote and edited the manuscript.

\section{Acknowledgments}

We thank M. Tse for her technical assistance. We acknowledge the Snyder Translational Laboratory in Critical Care Medicine for their research support in multiplexed bead array and analysis. We acknowledge the Alberta Sepsis Network for their financial support. We acknowledge the Critical Care Epidemiological and Biological Tissue Resource tissue bank for collecting, processing, managing the human samples. We acknowledge the Experimental Imaging Centre, Cumming School of Medicine, University of Calgary, for the assistance with the MRI imaging. We acknowledge the Live Cell Imaging Facility and Mouse Phenomics Resource Laboratory, both funded by the Snyder Institute, Cumming School of Medicine, University of Calgary. We acknowledge the Research Unit in Neurosciences, Cumming School of Medicine, University of Calgary. Finally, we would like to thank members of the Department of Critical Care Medicine, Cumming School of Medicine, University of Calgary, for recruiting and managing the patients with sepsis and, most importantly, the patients who participated in this research for without them we could not do this translational research.

Address correspondence to: Paul Kubes, HRIC 4AA16, 3330 Hospital Drive NW, Cumming School of Medicine, University of Calgary, Calgary, Alberta, T2N 4N1, Canada. Phone: 403.220.8558; Email: pkubes@ucalgary.ca. Or to: Christopher J. Doig, FMC-McCaig tower, Room 0449, 3134 Hospital Drive NW, Calgary, Alberta T2N 5A1, Canada. Phone: 403.944.1691; Email: cdoig@ucalgary.ca. Or to: Robert J. Sutherland. Canadian Centre for Behavioural Neuroscience, University of Lethbridge, 4401 University Drive, Lethbridge, Alberta T1K 3M4, USA. Phone: 403.394.3900; Email: robert.sutherland@uleth.ca.

1. Singer M, et al. The third international consensus definitions for sepsis and septic shock (Sepsis-3). JAMA. 2016;315(8):801-810.

2. In Focus: A National Look at Sepsis, Canadian Institute for Health Information. https://secure.cihi.ca/estore/productFamily. htm?locale=en\&pf=PFC1564. Published December 10, 2009. Accessed April 16, 2018.

3. Dremsizov T, Clermont G, Kellum JA, Kalassian KG, Fine MJ, Angus DC. Severe sepsis in community-acquired pneumonia: when does it happen, and do systemic inflammatory response syndrome criteria help predict course? Chest. 2006;129(4):968-978.

4. Lynch JP, Zhanel GG. Streptococcus pneumoniae: epidemiology, risk factors, and strategies for prevention. Semin Respir Crit Care Med. 2009;30(2):189-209.

5. File TM. Streptococcus pneumoniae and community-acquired pneumonia: a cause for concern. Am J Med. 2004;117 Suppl 3A:39S-50S.

6. Kaukonen KM, Bailey M, Suzuki S, Pilcher D, Bellomo R. Mortality related to severe sepsis and septic shock among critically ill patients in Australia and New Zealand, 2000-2012. JAMA. 2014;311(13):1308-1316.

7. Young GB. Encephalopathy of infection and systemic inflammation. J Clin Neurophysiol. 2013;30(5):454-461.

8. Gordon SM, Jackson JC, Ely EW, Burger C, Hopkins RO. Clinical identification of cognitive impairment in ICU survivors: insights for intensivists. Intensive Care Med. 2004;30(11):1997-2008. 
9. Hopkins RO, Weaver LK, Pope D, Orme JF, Bigler ED, Larson-LOHR V. Neuropsychological sequelae and impaired health status in survivors of severe acute respiratory distress syndrome. Am J Respir Crit Care Med. 1999;160(1):50-56.

10. Hopkins RO, Weaver LK, Collingridge D, Parkinson RB, Chan KJ, Orme JF. Two-year cognitive, emotional, and quality-of-life outcomes in acute respiratory distress syndrome. Am J Respir Crit Care Med. 2005;171(4):340-347.

11. Hough CL, Curtis JR. Long-term sequelae of critical illness: memories and health-related quality of life. Crit Care. 2005;9(2):145-146.

12. Yipp BG, Kubes P. NETosis: how vital is it? Blood. 2013;122(16):2784-2794.

13. Andonegui G, et al. Endothelium-derived Toll-like receptor-4 is the key molecule in LPS-induced neutrophil sequestration into lungs. J Clin Invest. 2003;111(7):1011-1020.

14. Springer TA. Traffic signals on endothelium for lymphocyte recirculation and leukocyte emigration. Annu Rev Physiol. 1995;57:827-872.

15. Petri B, et al. Endothelial LSP1 is involved in endothelial dome formation, minimizing vascular permeability changes during neutrophil transmigration in vivo. Blood. 2011;117(3):942-952.

16. Dal-Secco D, et al. A dynamic spectrum of monocytes arising from the in situ reprogramming of CCR $2+$ monocytes at a site of sterile injury. J Exp Med. 2015;212(4):447-456.

17. Yang J, Zhang L, Yu C, Yang XF, Wang H. Monocyte and macrophage differentiation: circulation inflammatory monocyte as biomarker for inflammatory diseases. Biomark Res. 2014;2(1):1.

18. Geissmann F, Manz MG, Jung S, Sieweke MH, Merad M, Ley K. Development of monocytes, macrophages, and dendritic cells. Science. 2010;327(5966):656-661.

19. Andonegui G, Goring K, Liu D, McCafferty DM, Winston BW. Characterization of S. pneumoniae pneumonia-induced multiple organ dysfunction syndrome: an experimental mouse model of gram-positive sepsis. Shock. 2009;31(4):423-428.

20. Banks WA, Kastin AJ, Broadwell RD. Passage of cytokines across the blood-brain barrier. Neuroimmunomodulation. 1995;2(4):241-248.

21. Skelly DT, Hennessy E, Dansereau MA, Cunningham C. A systematic analysis of the peripheral and CNS effects of systemic LPS, IL-1 $\beta$, [corrected] TNF- $\alpha$ and IL-6 challenges in C57BL/6 mice. PLoS One. 2013;8(7):e69123.

22. Cushman JD, Moore MD, Jacobs NS, Olsen RW, Fanselow MS. Behavioral pharmacogenetic analysis on the role of the $\alpha 4$ GABA(A) receptor subunit in the ethanol-mediated impairment of hippocampus-dependent contextual learning. Alcohol Clin Exp Res. 2011;35(11):1948-1959.

23. Saxe MD, et al. Ablation of hippocampal neurogenesis impairs contextual fear conditioning and synaptic plasticity in the dentate gyrus. Proc Natl Acad Sci USA. 2006;103(46):17501-17506.

24. Cunningham C. Microglia and neurodegeneration: the role of systemic inflammation. Glia. 2013;61(1):71-90.

25. Davalos D, et al. ATP mediates rapid microglial response to local brain injury in vivo. Nat Neurosci. 2005;8(6):752-758

26. Kozlowski C, Weimer RM. An automated method to quantify microglia morphology and application to monitor activation state longitudinally in vivo. PLoS One. 2012;7(2):e31814.

27. Sedgwick JD, Schwender S, Imrich H, Dörries R, Butcher GW, ter Meulen V. Isolation and direct characterization of resident microglial cells from the normal and inflamed central nervous system. Proc Natl Acad Sci USA. 1991;88(16):7438-7442.

28. D'Mello C, et al. Probiotics improve inflammation-associated sickness behavior by altering communication between the peripheral immune system and the brain. $J$ Neurosci. 2015;35(30):10821-10830.

29. Gofton TE, Young GB. Sepsis-associated encephalopathy. Nat Rev Neurol. 2012;8(10):557-566.

30. Sharshar T, et al. Brain lesions in septic shock: a magnetic resonance imaging study. Intensive Care Med. 2007;33(5):798-806.

31. McDonald B, et al. Intravascular danger signals guide neutrophils to sites of sterile inflammation. Science. 2010;330(6002):362-366.

32. Harding MG, Zhang K, Conly J, Kubes P. Neutrophil crawling in capillaries; a novel immune response to Staphylococcus aureus. PLoS Pathog. 2014;10(10):e1004379.

33. Rossaint J, et al. FGF23 signaling impairs neutrophil recruitment and host defense during CKD. J Clin Invest. 2016;126(3):962-974

34. Mizutani M, Pino PA, Saederup N, Charo IF, Ransohoff RM, Cardona AE. The fractalkine receptor but not CCR2 is present on microglia from embryonic development throughout adulthood. J Immunol. 2012;188(1):29-36

35. Singer $\mathrm{BH}$, et al. Cecal ligation and puncture results in long-term central nervous system myeloid inflammation. PLoS ONE. 2016;11(2):e0149136.

36. Morganti JM, et al. CCR2 antagonism alters brain macrophage polarization and ameliorates cognitive dysfunction induced by traumatic brain injury. $J$ Neurosci. 2015;35(2):748-760.

37. Hammond MD, et al. CCR2+ Ly6C(hi) inflammatory monocyte recruitment exacerbates acute disability following intracerebral hemorrhage. J Neurosci. 2014;34(11):3901-3909.

38. Varvel NH, et al. Infiltrating monocytes promote brain inflammation and exacerbate neuronal damage after status epilepticus Proc Natl Acad Sci USA. 2016;113(38):E5665-E5674

39. Yamasaki R, et al. Differential roles of microglia and monocytes in the inflamed central nervous system. J Exp Med. 2014;211(8):1533-1549.

40. Neves G, Cooke SF, Bliss TV. Synaptic plasticity, memory and the hippocampus: a neural network approach to causality. Nat Rev Neurosci. 2008;9(1):65-75

41. Squire LR. Memory and the hippocampus: a synthesis from findings with rats, monkeys, and humans. Psychol Rev . 1992;99(2):195-231.

42. Moser E, Moser MB, Andersen P. Spatial learning impairment parallels the magnitude of dorsal hippocampal lesions, but is hardly present following ventral lesions. J Neurosci. 1993;13(9):3916-3925.

43. Broadbent NJ, Squire LR, Clark RE. Spatial memory, recognition memory, and the hippocampus. Proc Natl Acad Sci USA. 2004;101(40):14515-14520.

44. Anagnostaras SG, Maren S, Sage JR, Goodrich S, Fanselow MS. Scopolamine and Pavlovian fear conditioning in rats: dose-effect analysis. Neuropsychopharmacology. 1999;21(6):731-744.

45. LeDoux JE, Cicchetti P, Xagoraris A, Romanski LM. The lateral amygdaloid nucleus: sensory interface of the amygdala in fear 
conditioning. J Neurosci. 1990;10(4):1062-1069.

46. Zenaro E, Piacentino G, Constantin G. The blood-brain barrier in Alzheimer's disease. Neurobiol Dis. 2017;107:41-56.

47. Fabis MJ, Scott GS, Kean RB, Koprowski H, Hooper DC. Loss of blood-brain barrier integrity in the spinal cord is common to experimental allergic encephalomyelitis in knockout mouse models. Proc Natl Acad Sci USA. 2007;104(13):5656-5661.

48. Zhou H, Andonegui G, Wong CH, Kubes P. Role of endothelial TLR4 for neutrophil recruitment into central nervous system microvessels in systemic inflammation. J Immunol. 2009;183(8):5244-5250.

49. Kolaczkowska E, Kubes P. Neutrophil recruitment and function in health and inflammation. Nat Rev Immunol. 2013;13(3):159-175.

50. Geissmann F, Jung S, Littman DR. Blood monocytes consist of two principal subsets with distinct migratory properties. Immunity. 2003;19(1):71-82.

51. D'Mello C, Le T, Swain MG. Cerebral microglia recruit monocytes into the brain in response to tumor necrosis factoralpha signaling during peripheral organ inflammation. J Neurosci. 2009;29(7):2089-2102.

52. D'Mello C, et al. P-selectin-mediated monocyte-cerebral endothelium adhesive interactions link peripheral organ inflammation to sickness behaviors. J Neurosci. 2013;33(37):14878-14888.

53. Liu L, Duff K. A technique for serial collection of cerebrospinal fluid from the cisterna magna in mouse. $J$ Vis Exp. 2008;(21):960

54. DeFries JC, Hegmann JP, Weir MW. Open-field behavior in mice: evidence for a major gene effect mediated by the visual system. Science. 1966;154(3756):1577-1579.

55. Dellu F, Contarino A, Simon H, Koob GF, Gold LH. Genetic differences in response to novelty and spatial memory using a two-trial recognition task in mice. Neurobiol Learn Mem. 2000;73(1):31-48. 